\title{
Coordinated Multiple Access Point Multiuser Beamforming Training Protocol for Millimeter Wave WLANs
}

\author{
Li-Hsiang Shen, Student Member, IEEE, Kai-Ten Feng, Senior Member, IEEE, Lajos Hanzo, Fellow, IEEE
}

\begin{abstract}
Millimeter wave (mmWave) systems have attracted considerable attention as a benefit of their Gbps services. Therefore, wireless-gigabit (WiGig) transmission has been developed based on the IEEE 802.11ad and 802.11ay standards of wireless local area networks (WLANs) in the 60-GHz band. To overcome the high path loss of mmWave, beamforming and beam-sector based training protocols are specified in 802.11ad/ay. With the increase in service demand, more WiGig access points (APs) will be deployed, hence the transmission frame structure of beam control should be adjustable for supporting both multiple APs and multiple users. The conventional exhaustive beam search (EBS) scheme has a potentially excessive complexity for high number of beams. Furthermore, assigning fixed training slots for users will lead to high-latency beam training under EBS. Hence, we design an advanced protocol for multiple APs and multiple users. Based on this protocol, we propose coordinated multiple AP multiuser training (CMMBT) including variable length framing and adjustable beam training. Our simulations demonstrate that CMMBT provides flexible training control and achieves the lowest training latency and highest average throughput performance amongst the state-of-the-art solutions in the open literature.
\end{abstract}

Index Terms-Millimeter wave communications, wireless local area networks, multi-user association, beamforming training, non-slotted frame structure.

\section{INTRODUCTION}

The increasing number of wireless devices and the rapid evolution of high-rate applications such as high definition video streaming have led to a considerable increase in the amount of wireless tele-traffic [1]. This issue is critical both for industry and academia, since the existing sub-6 GHz communication systems are simply unable to support the simultaneous high-rate service demands of a large number of connections. Hence, millimeter wave (mmWave) solutions having high spectral resources have attracted increasing attention in the context of next-generation systems [2]. Therefore, several international organizations are developing mmWave

Copyright (c) 2015 IEEE. Personal use of this material is permitted. However, permission to use this material for any other purposes must be obtained from the IEEE by sending a request to pubs-permissions@ieee.org.

L. Hanzo would like to acknowledge the financial support of the Engineering and Physical Sciences Research Council projects EP/N004558/1, EP/P034284/1, EP/P034284/1, EP/P003990/1 (COALESCE), of the Roya Society's Global Challenges Research Fund Grant as well as of the European Research Council's Advanced Fellow Grant QuantCom. K.-T. Feng would like to acknowledge the financial support of Ministry of Science and Technology (MoST) Grants 107-2221-E-009-058-MY3, 109-2218-E-009-002, Higher Education Sprout Project of the National Chiao Tung University and Ministry of Education (MoE), Taiwan. (Corresponding Authors: Kai-Ten Feng and Lajos Hanzo)

Li-Hsiang Shen and Kai-Ten Feng are with the Department of Electrical and Computer Engineering, National Chiao Tung University, Hsinchu, Taiwan. (email: gp3xu4vu6.cm04g@nctu.edu.tw; ktfeng@mail.nctu.edu.tw;)

Lajos Hanzo is with the Department of Electronics and Computer Science, University of Southampton, Southampton SO17 1BJ, U.K. (email: lh@ecs.soton.ac.uk) transmission protocols and techniques. For example, the thirdgeneration partnership project (3GPP) drafted new radio specifications for joint sub- $6 \mathrm{GHz}$ bands and mmWave bands under licensed spectrum utilization, especially at 28, 39, and 60 $\mathrm{GHz}$ carrier frequencies [3]. As for the unlicensed mmWave frequency bands, IEEE 802.11ad [4] and IEEE 802.11ay [5] have standardized $60-\mathrm{GHz}$ mmWave scenarios, requirements and transmission protocols for wireless local area networks (WLANs). Moreover, the wireless-gigabit (WiGig) initiative develops commercialized $60-\mathrm{GHz}$ solutions for high-rate transmission in addition to the mmWave WLAN protocols [6], [7].

Although mmWaves have the potential of supporting tensof-Gbps transmission rates, they suffer from high pathloss, penetration loss and atmospheric attenuation [8]-[10]. Hence, efficient analog/digital/hybrid beamforming designs have been proposed for supporting beam-based high-gain directional transmission for improving the coverage [10]-[14]. The analog beamformer adjusts the steering vectors, while the baseband digital beamformer further improves the gain by relying on several radio-frequency (RF) chains. Hybrid beamforming relying on both analog and digital beamformers strikes a compelling performance of cost/complexity trade-off [13], [14]. However, in contrast to conventional omni-directional communication at sub- $6 \mathrm{GHz}$ frequencies, each mobile device has to be pre-trained for finding the optimum beam direction. The associated beam sector based beamforming training mechanism of unlicensed mmWave solutions is specified by the IEEE 802.11ad/ay standards [15]. The central controller initiates a series of training procedures exchanging information between the transmitter and receiver, returning their corresponding beam directions [9]. Conventionally, exhaustive beam search (EBS) has been adopted for acquiring the complete set of signal-strength and beam-direction estimates [4], [16]. However, the traditional EBS scheme requires considerable time for beam training, leading to potentially excessive system overhead. A previous study elaborated on the trade-off between the operating beamwidth and latency/throughput [17], [18]. For transmissions associated with narrower beams, the EBS scheme results in increased latency and reduced throughput, which makes it less attractive, especially for numerous users and APs.

Hence, we continue by comparing our proposed beamforming training scheme relying on a beneficial frame structure to the most pertinent contributions in TableI . The majority of the existing literature has aimed for achieving low-latency beam training using either formal or heuristic optimization

Some abbreviations in Table I are as follows: Archite. = Architecture, $\mathrm{AP}=$ Access Point, UE $=$ User Equipment, Spec. Compata. $=$ Specification Compatibility, Associa. $=$ Association, Throu. $=$ Throughput, PS $=$ Partial Search, Req. = Requirement 
TABLE I

Comparison of Proposed Scheme with Existing Methods

\begin{tabular}{l|ccccccccc}
\hline Literature & Archite. & $\begin{array}{c}\text { Beam Training } \\
\text { AP-UE }\end{array}$ & $\begin{array}{c}\text { Spec. } \\
\text { Compata. }\end{array}$ & $\begin{array}{c}\text { Historical } \\
\text { Data }\end{array}$ & $\begin{array}{c}\text { Flexible } \\
\text { Structure }\end{array}$ & Associa. & $\begin{array}{c}\text { Beam } \\
\text { Alignment }\end{array}$ & Latency & Throu. \\
\hline \hline$[16]-[18]$ & P2P & EBS-EBS & $\checkmark$ & & & & & $\checkmark$ & $\checkmark$ \\
{$[19]$} & P2P & PS-PS & & & & & & $\checkmark$ & $\checkmark$ \\
{$[20],[21]$} & P2P & PS-PS & $\checkmark$ & & & & $\checkmark$ & $\checkmark$ & \\
{$[22]$} & P2P & EBS-PS & $\checkmark$ & $\checkmark$ & & & $\checkmark$ & $\checkmark$ & $\checkmark$ \\
{$[23],[24]$} & P2MP & EBS-EBS & & & & & $\checkmark$ & $\checkmark$ & \\
{$[25]$} & P2MP & EBS-EBS & $\checkmark$ & & & $\checkmark$ & & $\checkmark$ & $\checkmark$ \\
{$[26]$} & P2MP & EBS-EBS & $\checkmark$ & & & $\checkmark$ & & & \\
{$[27]$} & P2MP & EBS-PS & $\checkmark$ & $\checkmark$ & & $\checkmark$ & & $\checkmark$ & $\checkmark$ \\
{$[28]$} & P2MP & PS-PS & $\checkmark$ & & $\checkmark$ & $\checkmark$ & & $\checkmark$ & $\checkmark$ \\
{$[29],[30]$} & MP2MP & WiFi Req. & & & & & $\checkmark$ & $\checkmark$ & $\checkmark$ \\
{$[31],[32]$} & MP2MP & EBS-EBS & $\checkmark$ & & & & & $\checkmark$ & $\checkmark$ \\
Our Work & MP2MP & PS-PS & $\checkmark$ & $\checkmark$ & $\checkmark$ & $\checkmark$ & $\checkmark$ & $\checkmark$ & $\checkmark$ \\
\hline
\end{tabular}

schemes [19]-[22], mainly designed for point-to-point (P2P) transmissions. By contrast, the proposed training schemes are designed for low-complexity partial search (PS) relying on fewer training beams. They improve the performance by reducing the training length of beams by beneficially exploiting the correlated historical dataset or channel information. However, when supporting numerous directionally transmitting devices demanding high-rate services, the system overhead will increase because of the complex beam training process [4], [5]. For point-to-multi-point (P2MP) scenarios, sophisticated multiuser association and beam control should be designed for improving the conventional packet contention and exhaustive beam training mechanisms [23]-[28]. The system will result in degraded throughput upon using the EBS scheme for a high number of beams or users, which has not been considered in [23], [24]. In [25], the actively transmitting or receiving devices were exploited for reporting the idle or busy state of training slots, but the devices performed conventional EBS and random packet contention. The authors of [26] improved the contention mechanism by redesigning the backoff methods used for avoiding repeated packet collisions, albeit at the cost of introducing potentially excessive training overheads. Although the authors of [27], [28] have addressed the above-mentioned problems, they adopted a rigid fixed framing structure without considering the beam alignment outage probability. A further limitation of the existing studies is the consideration of a single access point (AP), which has limited performance in terms of serving a limited number of users. In more realistic networks, multiple mmWave base stations (BSs) or WiGig APs have to be established to construct a multi-point-to-multi-point (MP2MP) network for supporting numerous data transmission sessions at high traffic rates. In [29], [30], WiGig transmissions were ingeniously supported by learning from WiFi signals gleaned from other APs operating in lower frequency bands. However, they all require complex dual-band control mechanisms and extra signal measurements relying on several devices. In [31], [32], time-division based transmission structures were proposed for multiple WiGig APs and for multiple users employing the EBS method, which however require excessive time to train beams for numerous WiGig users. Moreover, the fixed framing design is not well suited for multiuser beam training.
Therefore, multi-AP multiuser beam training has not been addressed in the open literature. Hence, the WiGig protocol of multiple APs and multiple users has to be redesigned, whilst maintaining backwards compatibility with the current standards for achieving an improved system performance. Our contributions are summarized as follows.

- We conceive the first WiGig based beam transmission frame structure for multiple WiGig APs and multiple users considering both user association and beamforming training mechanisms. The proposed protocols are also backward-compatible with the existing unlicensed IEEE 802.11ad/ay standards.

- $\quad$ Our research objective is to maximize the average throughput constrained both by the successful user association ratio and the maximum tolerable beam alignment outage probability. We proposed an AP coordination mechanism, which is decomposed into a pair of sub-problems considering the WiGig controller/APs and the WiGig users, respectively. The WiGig APs aim for flexibly adjusting the length of both the training frame and contention slots, while the user association and beam training policy are determined by the WiGig users. We simultaneously consider both the WiGig system's framing and the AP/user beam training, which has not been jointly considered in the existing studies.

- We evaluate the system performance attained with a ray-tracing based emulator for realistic IEEE 802.11ad/ay wireless environments. We quantify the user association ratio, beam alignment outage probability, training latency and system throughput. The proposed WiGig based AP/user coordination mechanism outperforms the existing techniques both in terms of its latency and throughput.

We commence by introducing the proposed WiGig based coordinated multi-AP multiuser frame structure and our beam training protocols in Section II-A. In Section II-B, we design the system models and formulate our optimization problem by maximizing the throughput, while additionally considering both the successful association ratio and beam alignment accuracy. We continue by proposing a coordinated multi-AP multiuser beam training scheme in Section III. In Section IV, 
we first discuss the relationship between the parameters and the metrics of beam alignment accuracy, latency and throughput. We then evaluate our proposed scheme using a ray-tracing based emulator and compare conventional schemes. Finally, we conclude in Section V.

\section{Frame Structure and Proposed System}

\section{A. Multi-AP Multiuser Frame Structure}

The WiGig system supports high-speed mmWave transmission in the 60-GHz frequency band, which is developed based on the IEEE 802.11ad/ay standards. The new periodic frame structure proposed for multi-AP multiuser beamforming training followed by the IEEE $802.11 \mathrm{ad} / \mathrm{ay}$ protocol is shown in Fig. 1, which relies on the so-called beacon interval (BI) [33]. A typical BI includes the time duration of the control overhead, termed as the beacon header interval (BHI) which is followed by a data transmission interval (DTI). In the improved framing proposed, several WiGig APs are regarded as distributed units connected to the central unit, namely to the WiGig controller. This controller is responsible for centrally coordinating multiple WiGig APs for consecutively starting their initial beam training. Moreover, the beam control mechanism and network information exchange taking place during a BHI contain three sub-intervals, namely the beacon transmission interval (BTI), the association and beamforming training (A-BFT), and the announcement transmission interval (ATI).

The overall signaling protocol of the proposed beam-based control and transmission is shown in Fig. 2. We proposed to design and extend the conventional 802.11ad/ay single-AP protocol to a multi-AP multiuser scenario, which additionally requires control management and consecutive time-division based training sub-intervals. First, the WiGig controller and APs execute synchronization and exchange the parameters required for beam control management at the beginning of each $\mathrm{BI}$, which is not defined in original 802.11ad/ay protocols. Based on the signaling of Fig. 2, the group of APs train their beam sectors with 802.11 ad/ay packets within multiple directional beacon frames during the beaconing session of BTI. The beacon announcement is delivered to all listening WiGig users for all possible beam directions. The transmitter will conduct directional training, and the receiver listens either omni- or quasi-directionally. In the A-BFT subframe, several training A-BFT slots containing consecutive sector sweep (SSW) frames are provided by the WiGig APs for the users in order to simultaneously train their antenna sectors and to become associated with the desired AP. The users have to contend during the A-BFT slots for training opportunities. As a result, the candidate beam information of the APs and of the users is transmitted during the SSWs from the users and by providing feedback (FB) from the AP side, respectively. As seen in Fig. 2, after completion of contention by the users during A-BFT, the FB and acknowledgement (ACK) packets are exchanged between the WiGig APs and users. Additionally, the WiGig APs and users will exchange the association and transmission control information in the ATI. The WiGig controller will determine its allocation policy for user association and beam assignment of WiGig APs as
TABLE II

DEFINITION OF SYSTEM PARAMETERS

\begin{tabular}{|l|l|}
\hline Parameters & Notation \\
\hline \hline Number of WiGig APs & $N$ \\
\hline Number of WiGig users & $K$ \\
\hline Beam set of WiGig APs / users & $\boldsymbol{\Phi}_{n}^{A P} / \boldsymbol{\Phi}_{k}^{U E}$ \\
\hline Number of AP / user beam sectors & $L_{n}^{A P} / L_{k}^{U E}$ \\
\hline Candidate AP / user beam training set & $\phi_{n}^{A P}(t) / \phi_{k}^{U E}(t)$ \\
\hline Number of AP / user training beams & $L_{n}^{A P}(t) / L_{k}^{U E}(t)$ \\
\hline SNR of AP / user training beams & $\gamma_{n, k}^{A P}(t) / \gamma_{n, k}^{U E} l_{k}(t)$ \\
\hline Transmitted training power of AP / user & $P_{t}^{A P} / P_{t}^{U E}$ \\
\hline Beam gain model & $G_{(\theta)}$ \\
\hline Transmit beam direction of AP / user & $\theta \in\left\{\theta_{n, l_{n}}, \theta_{k, l_{k}}\right\}$ \\
\hline Half-power beamwidth & $\theta_{-3 d B}$ \\
\hline Channel of WiGig APs / users & $H_{n, k, l_{n}}(t) / H_{n, k, l_{k}}(t)$ \\
\hline Noise power spectral density & $N_{0}$ \\
\hline WiGig system bandwidth & $B$ \\
\hline Decodable power thresholds of APs / users & $\gamma_{d e c}^{A P} / \gamma_{d e c}^{U E}$ \\
\hline Provided / Maximum number of A-BFT slots & $M_{n}(t) / M_{n}$ \\
\hline Provided / Maximum number of SSW frames & $F_{n}(t) / F_{n}$ \\
\hline Contention policy of WiGig users & $s_{n, k}(t)$ \\
\hline Number of contending users & $\chi_{n, m}(t)$ \\
\hline Optimal training beam of APs / users & $l_{n, k}^{* A P}(t) / l_{n, k}^{* U E}(t)$ \\
\hline System beam training latency & $T_{t r}(t)$ \\
\hline Duration of BI / FB / ACK & $T_{B I} / T_{f b} / T_{a c k}$ \\
\hline Duration of each beam training & $T_{b m}$ \\
\hline SNR of downlink data transmission & $\gamma_{n, k, l}^{D L}(t)$ \\
\hline Downlink transmission power & $P_{t}^{D L}$ \\
\hline System throughput & $R(t)$ \\
\hline System successful association ratio & $S A(t)$ \\
\hline System beam alignment outage probability & $B O(t)$ \\
\hline Historical observation window size & $W_{\mathcal{A}}$ \\
\hline Threshold of beam alignment outage & $\delta_{b o}$ \\
\hline Threshold of successful association & $\delta_{s a}$ \\
\hline Short-term beam outage probability & $Q_{b o}(t)$ \\
\hline Short-term successful association ratio & $Q_{s a}(t)$ \\
\hline Portion of number of tuned parameters & $\lambda_{\mathcal{A}}$ \\
\hline Set of tuned parameters & \\
\hline Decreasing / Increasing portion function & $\lambda_{A}^{+}$ \\
\hline
\end{tabular}

demonstrated in Fig. 2, followed by downlink data transmission during DTI and the final session of reporting transmission results. The DTI contains multiple contention-based access periods (CBAPs) and scheduled service periods (SPs) for all APs and the users to transfer the desired data, respectively. The users contend for resources in CBAPs, and SPs are assigned by the controller for contention-free transmission. At the end of each BI, the controller stores all datasets and initiates the next BI

\section{B. System Model}

We consider $N$ WiGig APs coordinated by a single WiGig controller serving $K$ WiGig users in the network. For each WiGig AP and WiGig user, the beam set is given as $\boldsymbol{\Phi}_{n}^{A P}$ and $\boldsymbol{\Phi}_{k}^{U E}$, respectively, containing $L_{n}^{A P}$ and $L_{k}^{U E}$ number of beam sectors. In one slot, only one beam direction is utilized for transmitting/receiving signals based on the 802.11ad/ay protocols. During the $t$-th BI, both the WiGig APs and the users will determine their candidate beam training set of $\phi_{n}^{A P}(t)$ and $\phi_{k}^{U E}(t)$ having the corresponding length of $L_{n}^{A P}(t)$ and $L_{k}^{U E}(t)$, respectively. The candidate training set is a subset of the original beam set, i.e. we have $\phi_{n}^{A P}(t) \in \boldsymbol{\Phi}_{n}^{A P}$ and $\phi_{k}^{U E}(t) \in \boldsymbol{\Phi}_{k}^{U E}$, and the constraints on the length of candidate set can be further expressed as $L_{n}^{A P}(t) \leq L_{n}^{A P}$ and $L_{k}^{U E}(t) \leq L_{k}^{U E}$. Based on the protocol designed, WiGig users will receive consecutive training beacon packets from the APs during the BTI. The signal-to-noise-ratio (SNR) of the $n$-th 


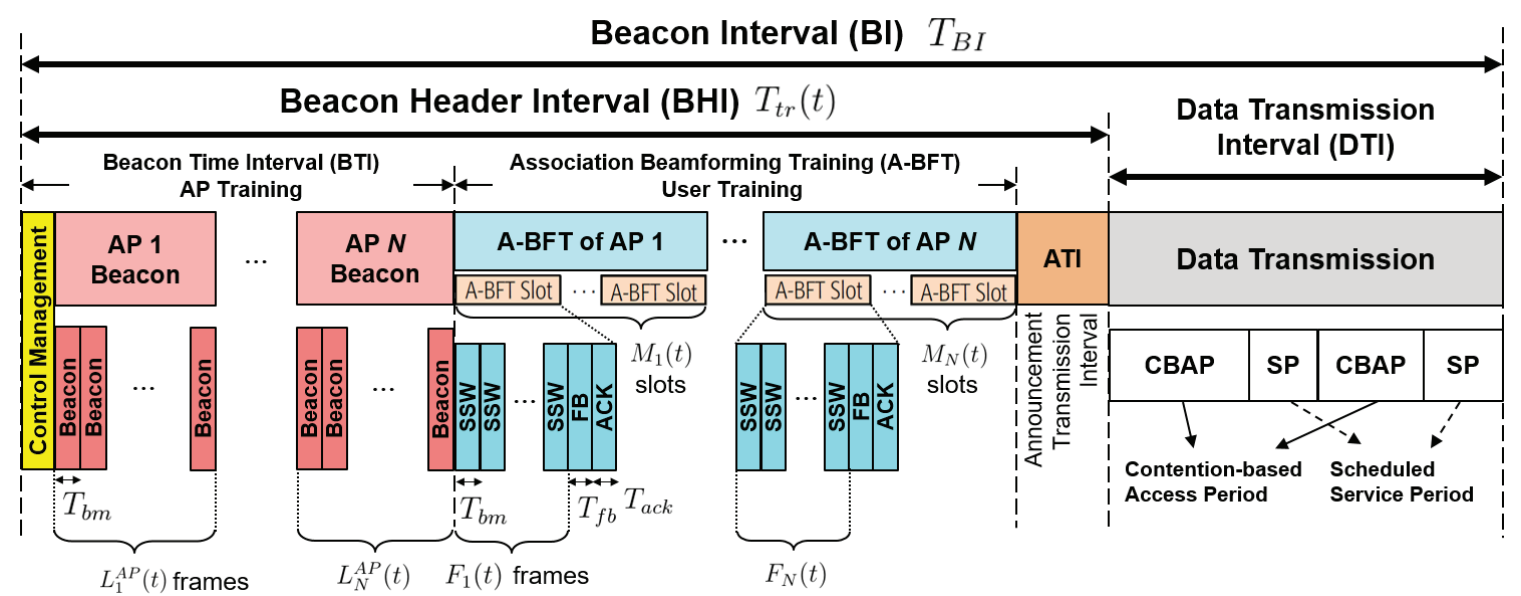

Fig. 1. Proposed frame structure for multi-AP multiuser transmission based on IEEE 802.11ad/ay protocols. Note that SSW/FB/ACK represent the sector sweep/feedback/acknowledgement packets, respectively.

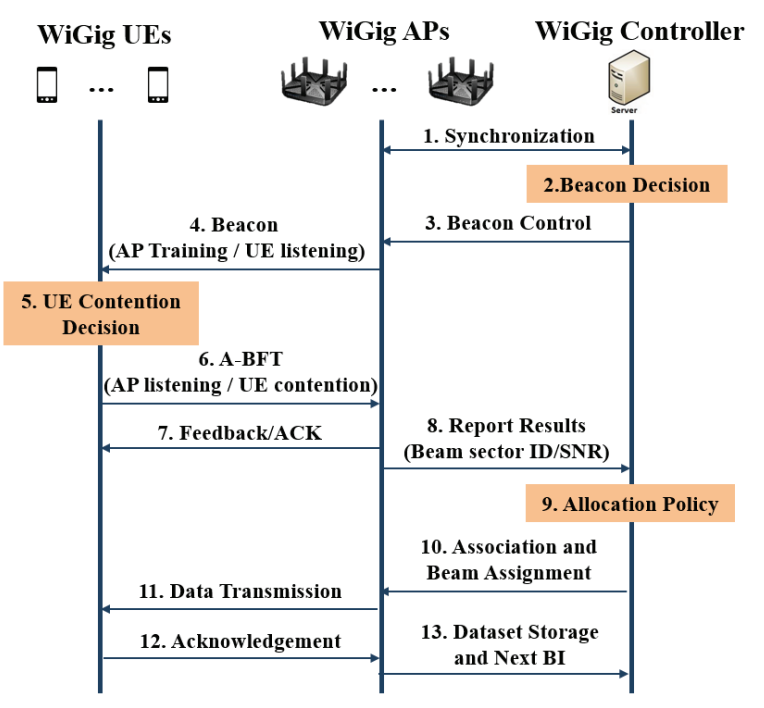

Fig. 2. Signaling coordinated beam control for multi-AP multiuser WiGig transmission.

WiGig AP at the $k$-th user's downlink receiver employing the $l_{n}$-th beam in the $t$-th BI is

$$
\gamma_{n, k, l_{n}}^{A P}(t)=\frac{P_{t}^{A P} G\left(\theta_{n, l_{n}}\right) H_{n, k, l_{n}}(t)}{N_{0} B},
$$

where $P_{t}^{A P}$ is the transmitted training power of APs, $G\left(\theta_{n, l_{n}}\right)$ is the beam gain associated with the transmit direction $\theta_{n, l_{n}}$, and $H_{n, k, l_{n}}(t)$ is the downlink channel's transfer function between APs and users. The noise power is given by $N_{0} B$, where $N_{0}$ is the noise power spectral density, and $B$ is the WiGig system bandwidth. Similar to (1), the SNR of user beam training during A-BFT of the $k$-th WiGig user with respect to the $n$-th AP using the $l_{k}$-th beam can be written as $\gamma_{n, k, l_{k}}^{U E}(t)=\frac{P_{t}^{U E} G\left(\theta_{k, l_{k}}\right) H_{n, k, l_{k}}(t)}{N_{0} B}$, where $P_{t}^{U E}$ is the user's transmitted training power. The beam gain model is defined as an exponential expression based on the 802.11ad/ay specifications [34], which is given by

$$
G(\theta)=G_{0} e^{-\alpha \theta^{2}}
$$

where $G_{0}$ is the maximum configured antenna gain, $\theta=$ $\left\{\theta_{n, l_{n}}, \theta_{k, l_{k}}\right\}$ is the transmit direction of each beam sector, and $\alpha$ is a constant coefficient determined by the half-power beamwidth (HPBW) $\theta_{-3 d B}$ as $\alpha=\frac{4 \ln (2)}{\theta^{2}}$ [34]. Without loss of generality, the received signals are decodable, when they are higher than the pre-defined successful decoding power thresholds $\gamma_{\text {dec }}^{A P}$ and $\gamma_{d e c}^{U E}$ for the AP and user training, respectively, i.e., when we have $\gamma_{n, k, l_{n}}^{A P}(t) \geq \gamma_{d e c}^{A P}$ and $\gamma_{n, k, l_{k}}^{U E}(t) \geq \gamma_{d e c}^{U E}$. Naturally, the signal strengths of the training beams become weaker both for reflected and for blocked channel paths, which potentially leads to undecodable reception.

During A-BFT, each WiGig AP will provide $M_{n}(t)$ ABFT slots for the WiGig users' contention, where we have $1 \leq M_{n}(t) \leq M_{n}$ and $M_{n}$ is the maximum affordable number of A-BFT slots. In each A-BFT slot, $F_{n}(t)$ SSW frames are offered to the users for their beam training. Explicitly, the number of frames provided must not exceed the maximum number of beams of the WiGig users, i.e., we have $F_{n}(t) \leq F_{n} \triangleq \max _{k} L_{k}^{U E}$. We define $\mathcal{S}=$ $\left\{s_{n, k}(t) \mid \forall 1 \leq n \leq N, 1 \leq k \leq K\right\}$ as the contention policy of users, implying that the $k$-th WiGig user intends to associate with the $n$-th WiGig AP at the $\left(s_{n, k}(t)\right)$-th A-BFT slot. For example, $s_{1,2}(t)=3$ indicates that the second user contends for the third A-BFT slot of AP 1. Therefore, we can obtain the total number of contentions $\chi_{n, m}(t)$ for the $n$-th $\mathrm{AP}$ at the $m$-th A-BFT slot given by

$$
\chi_{n, m}(t)=\sum_{k=1}^{K} \mathbb{1}\left(s_{n, k}(t)=m\right),
$$

where $\mathbb{1}(\cdot)$ is the indicator function. When completing the training processes, we obtain the indices of best beams relying on the optimum SNR reports of the WiGig APs/users, which are respectively given as

$$
\begin{aligned}
& l_{n, k}^{* A P}(t)=\underset{l_{n} \in \phi_{n}(t)}{\operatorname{argmax}} \gamma_{n, k, l_{n}}^{A P}(t), \\
& l_{n, k}^{* U E}(t)=\underset{l_{k} \in \phi_{k}(t)}{\operatorname{argmax}} \gamma_{n, k, l_{k}}^{U E}(t) \mathbb{1}\left(\chi_{n, s_{n, k}}(t)=1\right) .
\end{aligned}
$$

The best beams of WiGig users in (4b) are determined by both the optimum SNR and the successful contention defined 
in (3). During DTI, all WiGig APs and WiGig users will adopt the beams $l_{n, k}^{* A P}(t)$ and $l_{n, k}^{* U E}(t)$, respectively, for the desired transmissions.

We consider $T_{B I}$ as the time duration of the periodic BI, and $T_{b m}$ symbolizes the training overhead of each beam sector. We denote the duration of FB and ACK by $T_{f b}$ and $T_{a c k}$, respectively. Therefore, the overall latency imposed by the overhead of our WiGig based multi-AP multiuser framing at the $t$-th BI can be expressed as

$$
T_{t r}(t)=\sum_{n=1}^{N}\left[T_{b m} L_{n}^{A P}(t)+M_{n}(t)\left(T_{b m} F_{n}(t)+T_{f b}+T_{a c k}\right)\right] .
$$

It is worth mentioning that in (5), the first term of $T_{b m} L_{n}^{A P}(t)$ is responsible for the overhead of beam training information exchange from the WiGig APs and users at the BTI. On the other hand, the remaining term of $M_{n}(t)\left(T_{b m} F_{n}(t)+T_{f b}+T_{a c k}\right)$ is the duration of the A-BFT, during which the WiGig users transmit beam training information to the desired WiGig AP. The durations of control management and ATI are neglected because we mainly focus on attention on reducing the overhead of beamforming training. Here, we consider time-division access based downlink transmission, which means that there is no interference, and the corresponding SNR is defined as

$$
\gamma_{n, k, l^{*}}^{D L}(t)=\frac{P_{t}^{D L} G\left(\theta_{n, l_{n, k}^{* A P}(t)}\right) G\left(\theta_{k, l_{n, k}^{* U E}(t)}\right) H_{n, k, l^{*}}(t)}{N_{0} B},
$$

where $l^{*} \in\left\{l_{n, k}^{* A P}(t)\right.$ and $\left.l_{n, k}^{* U E}(t)\right\}$ is the optimum transmission beam set for the WiGig APs and users, while $P_{t}^{D L}$ is the downlink transmission power. Moreover, we can infer that user association is related to access contention policy $s_{n, k}(t)$ of the WiGig users, because the users can only be served when the packet contention is indeed successful. We can then obtain the average throughput by both considering training latency and the signal strength, which may be formulated as

$$
\begin{aligned}
R(t)=B \sum_{n=1}^{N} & \sum_{k=1}^{K} \max \left[0,1-\frac{T_{t r}(t)}{T_{B I}}\right] . \\
& \log _{2}\left(1+\gamma_{n, k, l^{*}}^{D L}(t)\right) \mathbb{1}\left(\chi_{n, s_{n, k}}(t)=1\right) .
\end{aligned}
$$

No data transmission will be executed if it would require excessive training overhead, i.e., $T_{t r}(t) \geq T_{B I}$. In general, successful contention occurs if there is only one WiGig user in the slot, i.e., $\chi_{n, m}(t)=1$. Based on (3), the successful association ratio $S A(t)$ can be expressed as

$$
S A(t)=\frac{1}{K} \sum_{n=1}^{N} \sum_{m=1}^{M_{n}(t)} \mathbb{1}\left(\chi_{n, m}(t)=1\right) .
$$

Furthermore, a beam alignment outage event $B O(t)$ occurs when signal strength of training from both WiGig AP $n$ and WiGig user $k$ required for their optimum beams falls belows its minimum, which is expressed as

$$
\begin{aligned}
B O(t)=\frac{1}{K} \sum_{k=1}^{K} \mathbb{1}\left(\gamma_{n, k, l_{n, k}^{* A P}}^{A P}(t) \leq \gamma_{t h}^{A P} \cap\right. \\
\left.\gamma_{n, k, l_{n, k}^{* U E}}^{U E}(t) \leq \gamma_{t h}^{U E} \mid n=\arg _{i} s_{i, k}(t)\right),
\end{aligned}
$$

where $\gamma_{t h}^{A P}$ and $\gamma_{t h}^{U E}$ are these predefined beam alignment thresholds and (9) is computed based on the WiGig user's association policy $s_{i, k}(t)$ with respect to the desired WiGig AP. The summary of system parameters and notations is illustrated in Table II.

\section{Problem Formulation}

It is beneficial to take into account the short-term history of the beam alignment and user association. Therefore, our problem is to maximize the average system throughput $R(t)$ by finding the most suitable AP and user training beam set $\phi_{n}^{A P}(t), \phi_{k}^{U E}(t)$, the length of A-BFT frames $F_{n}(t)$, the number of A-BFT slots $M_{n}(t)$ and contention policy $s_{n, k}(t)$ as

$$
\begin{array}{ll} 
& \max _{\phi_{n}^{A P}(t), \phi_{k}^{U E}(t), F_{n}(t), M_{n}(t), s_{n, k}(t)} R(t) \\
\text { s.t. } & \frac{1}{W+1} \sum_{\tau=0}^{W} B O(t-\tau) \leq \delta_{b o}, \\
& \frac{1}{W+1} \sum_{\tau=0}^{W} S A(t-\tau) \geq \delta_{s a} \\
& \phi_{n}^{A P}(t) \in \boldsymbol{\Phi}_{n}^{A P} \\
& \phi_{k}^{U E}(t) \in \boldsymbol{\Phi}_{k}^{U E} \\
& 1 \leq F_{n}(t) \leq F_{n} \\
& 1 \leq M_{n}(t) \leq M_{n} \\
& s_{n, k}(t) \in\left[1, M_{n}(t)\right] .
\end{array}
$$

The problem considered in (10) is constrained both by the short-term beam alignment outage events in (10b) and by the successful association ratio in (10c) within $W$ historical outcomes. We denote the thresholds of beam alignment accuracy and of the successful association ratio by $\delta_{b o}$ and $\delta_{s a}$, respectively. The constraints $(10 \mathrm{~d})$ and $(10 \mathrm{e})$ indicate that the size of the beam training index set cannot exceed the total size of beam index set. The constraints (10f) and (10g) are for maximum available SSW frames and A-BFT slots provided by the WiGig APs. In (10h), the association index is determined within the available number of A-BFT slots provided by APs. Moreover, we can see that (10) is non-convex and nonlinear due to the coupled computation of a maximum function, which depends both on the beam training overhead, as well as on a logarithmic function of the downlink SNR, and on an indicator function of the successful association. Hence, there does not exist appropriate transformations of the closed-form of the NP-hard mixed combinatorial problem in (10) due to the presence of coupled continuous and discrete variables, which

We consider fixed power and beamwidth configuration for WiGig beam training. Generally, having a flexible controllable transmission power and beamwidth was widely considered in the open literature in terms of a radio resource allocation problem, which typically takes place during downlink data transmission at the DTI. 


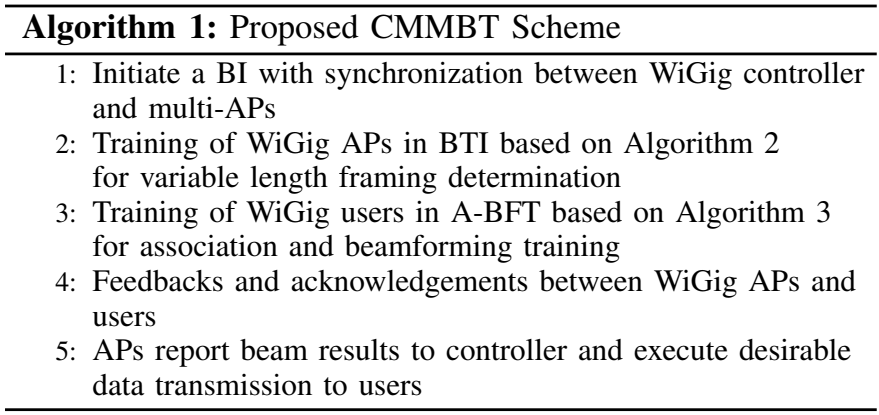

leads to open challenges in terms of deriving analytically solvable results. In the following, we propose a coordination mechanism for heuristically obtaining the candidate solution.

\section{Proposed Coordinated Multi-AP Multiuser BEAM TRAINING SCHEME}

Owing to its non-convexity and non-linearity, the original problem in (10) is decomposed into two parts, one for WiGig controller/APs and the other for WiGig users, where the respective arguments are $\left\{\phi_{n}^{A P}(t), F_{n}(t), M_{n}(t)\right\}$ and $\left\{\phi_{k}^{U E}(t), s_{n, k}(t)\right\}$. Therefore, we proposed a coordinated multi-AP multiuser beam training (CMMBT) scheme for variable length framing (VLF) for WiGig APs and adjustable beam training (ABT) for WiGig users. The overall CMMBT scheme is described in Algorithm 1. First, the WiGig controller initiates a BI and synchronizes all WiGig APs. Then, after the BTI, the WiGig APs train their beams using VLF in Algorithm 2 , where the controller obtains the historical training results from all APs to determine the beam training set $\phi_{n}^{A P}(t)$, the number of A-BFT slots $M_{n}(t)$, and the corresponding length of A-BFT frame $F_{n}(t)$. Furthermore, WiGig users adopt the ABT scheme of Algorithm 3 individually associated with the most desirable WiGig AP $n$ and determine the parameters of both the beam training set $\phi_{k}^{U E}(t)$ and of the contention policy $s_{n, k}(t)$. When completing all the training procedures, the associated FBs and ACKs are exchanged between WiGig APs and the users. The APs commence their directional data transmission during DTI following the training results. We will elaborate on the proposed VLF scheme conceived for the WiGig APs in the BTI and on the ABT method designed for WiGig users in A-BFT.

\section{A. Proposed VLF Scheme for WiGig APs in BTI}

The proposed VLF scheme is illustrated in Algorithm 2. We define a pair of parameters, one for short-term beam alignment outage probability and one for the successful association ratio formulated as $Q_{b o}(t)=\sum_{\tau=1}^{W} B O(t-\tau) /(W+1)$ and $Q_{s a}(t)=\sum_{\tau=1}^{W} S A(t-\tau) /(W+1)$, respectively. Note that they are slightly different from (10b) and (10c) because the training result at the $t$-th $\mathrm{BI}$ is undetermined, i.e., we have $\tau=0$. Experiencing beam alignment failure and user association failure as the lower bound of constraints in (10b) and (10c) is considered to be the worst case. As a result, we can re-formulate our problem for the WiGig controller and APs as

$$
\begin{array}{ll} 
& \max _{\phi_{n}^{A P}(t), F_{n}(t), M_{n}(t),} \quad R(t) \\
\text { s.t. } & Q_{b o}(t) \leq \delta_{b o}, \\
& Q_{s a}(t) \geq \delta_{s a}, \\
& (10 \mathrm{f}),(10 \mathrm{~g}) .
\end{array}
$$

Furthermore, we can observe that the constraint of beam alignment is uncorrelated with the successful user association ratio, which implies that the number of contention A-BFT slots provided is independent of that of the SSW frames. Explicitly, the number of A-BFT slots $M_{n}(t)$ is only related to the number of contending users $\chi_{n, m}(t)$, as shown in (8). However, only the receiver's SNR is considered in the beam alignment expression of (9), which is uncorrelated with the number of association slots provided. Therefore, the constraint (11b) is independent of (11c). In other words, the number of slots $M_{n}(t)$ will affect neither the beam policy $\phi_{n}^{A P}(t)$ nor the number of frames $F_{n}(t)$. The problem (11) can be separated into the problem of determining the beam training policy of WiGig APs

$$
\begin{aligned}
& \max _{\phi_{n}^{A P}(t), F_{n}(t)} R\left(t ; M_{n}(t)\right) \\
& \text { s.t. } \quad(10 \mathrm{f}),(11 \mathrm{~b}),
\end{aligned}
$$

and quantifying the number of A-BFT contention slots

$$
\begin{aligned}
& \max _{M_{n}(t)} R\left(t ; \phi_{n}^{A P}(t), F_{n}(t)\right) \\
& \text { s.t. }(10 \mathrm{~g}),(11 \mathrm{c}),
\end{aligned}
$$

in conjunction with the policies $\left\{\phi_{n}^{A P}(t), F_{n}(t)\right\}$ and $M_{n}(t)$, respectively. As for (12a), it indicates that we maximize the throughput given $M_{n}(t)$; finally (13a) represents the optimization given the results of $\phi_{n}^{A P}(t)$ and $F_{n}(t)$, respectively. Note that the WiGig users' policies of beam training $\phi_{k}^{U E}(t)$ and contention $s_{n, k}(t)$ are respectively constrained by WiGig parameters in (12) and (13), explicitly, we have $1 \leq\left|\phi_{k}^{U E}(t)\right| \leq F_{n}(t)$ and $1 \leq s_{n, k}(t) \leq M_{n}(t)$ if user $k$ attempts association to AP $n$. However, sub-problem (12) is unsolvable for beam indices $\phi_{n}^{A P}(t)$ because of the uncertainties of the users' behavior and owing to the complex environmental factors. Furthermore, for (13), the number of A-BFT slots $M_{n}(t)$ is affected by the user beam training and association policies, leading to unsolvable sub-problems.

Therefore, we proposed the VLF scheme for the WiGig controller coordinating the WiGig APs to flexibly adjust the length of the beam training frames, as detailed in Algorithm 2. First, we define the parameters to determine the required portion of training beams, SSW frames, and contention slots which are denoted as $\lambda_{\text {sec }} \triangleq \frac{L_{n}^{A P}(t)}{L_{n}^{A P}}, \lambda_{s s w} \triangleq \frac{F_{n}(t)}{F_{n}}$ and $\lambda_{\text {slot }} \triangleq \frac{M_{n}(t)}{M_{n}}$, respectively. We then define $\lambda_{\mathcal{A}}^{-}$as the policy for decreasing the portion and $\lambda_{\mathcal{A}}^{+}$as the one for increasing the portion of beams, frames and slots of $\lambda_{\mathcal{A}}$, where $\mathcal{A}=\{$ sec, ssw, slot $\}$ and we have $0<\lambda_{\mathcal{A}}^{-} \leq 1 \leq \lambda_{\mathcal{A}}^{+}<\infty$. Based on problem (12), when $Q_{b o}(t)$ is lower than the pre-defined threshold $\delta_{b o}$, the WiGig system attains a compellingly low beam alignment outage probability. The system will reduce WiGig APs' beam 


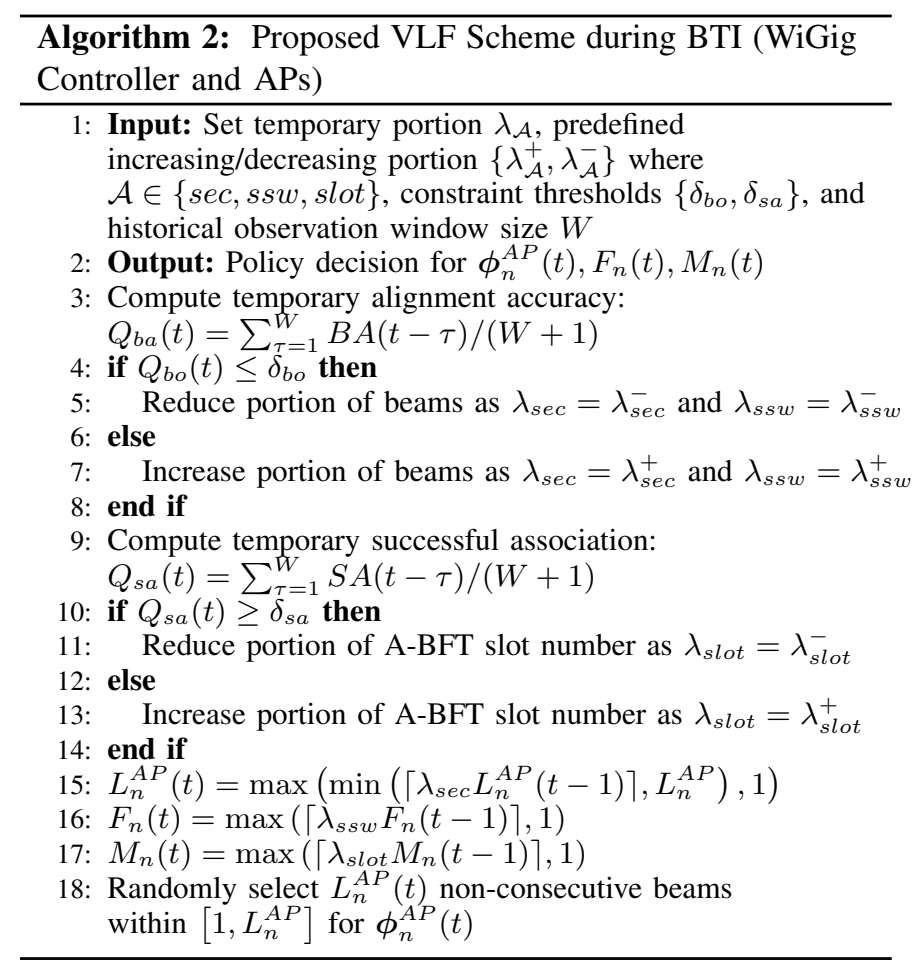

training portion of $\phi_{n}^{A P}(t)$ by $\lambda_{s e c}^{-}$and decrease the portion of SSW frames $F_{n}(t)$ in A-BFT by $\lambda_{s s w}^{-}$. Similarly, to satisfy the requirement in (11b), the WiGig controller maintains its performance by increasing the portion of training beams and SSW frames, i.e., we have $\lambda_{s e c}=\lambda_{s e c}^{+}$and $\lambda_{s s w}=\lambda_{s s w}^{+}$, respectively. Similarly, for (13), when $Q_{s a}(t)$ is higher than $\delta_{s a}$, the system reduces the portion of A-BFT slots provided for the WiGig users by $\lambda_{\text {slot }}^{-}$; however, it will increase the portion by $\lambda_{\text {slot }}^{+}$to satisfy the constraint (11c). In summary, the policy is then determined by the product of the corresponding portion and previous policy at $\mathrm{BI}(t-1)$, where the number of training beams of WiGig AP $L_{n}^{A P}(t)$, that of SSW frames in A-BFT provided for WiGig users $F_{n}(t)$, and that of A-BFT slots $M_{n}(t)$ can be, respectively, represented by

$$
\begin{aligned}
L_{n}^{A P}(t) & =\max \left(\min \left(\left\lceil\lambda_{\text {sec }} L_{n}^{A P}(t-1)\right\rceil, L_{n}^{A P}\right), 1\right), \\
F_{n}(t) & =\max \left(\left\lceil\lambda_{\text {ssw }} F_{n}(t-1)\right\rceil, 1\right), \\
M_{n}(t) & =\max \left(\left\lceil\lambda_{\text {slot }} M_{n}(t-1)\right\rceil, 1\right),
\end{aligned}
$$

where $\lceil\cdot\rceil$ is the ceiling operation acquiring integer variables. Additionally, all parameters are constrained by the maximum function owing to the non-negative values. Because of the random factors of the WiGig users and complex propagation environments, it is a challenge for the WiGig controller to coordinate APs where to transmit beams. As a result, after determining the training length $L_{n}^{A P}(t)$, the policy of beam indices of WiGig APs is selected randomly and uniformly for $\phi_{n}^{A P}(t) \in\left[1, L_{n}^{A P}\right]$, where $\left|\phi_{n}^{A P}(t)\right|=L_{n}^{A P}(t)$. For example, as depicted in Fig. 3, we consider the policy determination of the number of SSW frames $F_{n}(t)$ for a maximum available frame of $F_{n}=10$ for portions $\lambda_{s s w}^{-}=0.5$, and $\lambda_{s s w}^{+}=1.5$. If the beam alignment outage threshold is satisfied at the $(t-2)$-nd BI, the controller will shorten the SSW frames provided next time, i.e., we have $F_{n}(t-1)=\lambda_{s s w}^{-} F_{n}(t-2)=$

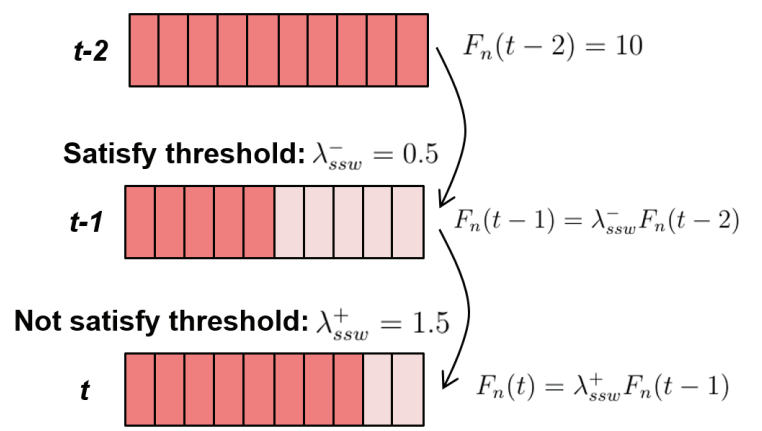

Fig. 3. Illustration of proposed VLF scheme. We consider the policy determination for the number of SSW frames $F_{n}(t)$ with maximum available frames $F_{n}=10$ and portions $\lambda_{s s w}^{-}=0.5$ and $\lambda_{s s w}^{+}=1.5$.

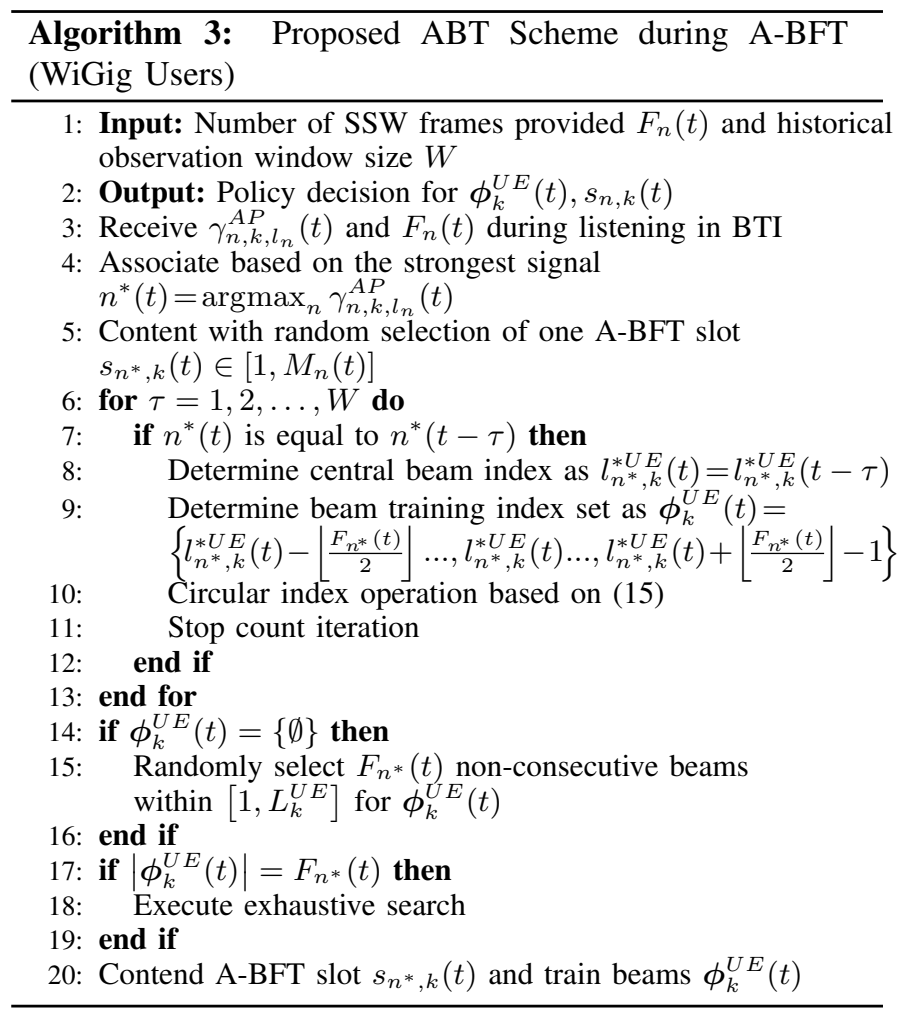

$0.5 \times 10=5$ frames. Otherwise, it increases the number of frames as $F_{n}(t)=\lambda_{s s w}^{+} F_{n}(t-1)=1.5 \times 5=7.5$. Note that the number of frames is an integer value; thus, it provides $F_{n}(t) \approx 8$ frames at time $t$. The same operation is performed for the number of training beams of WiGig APs $L_{n}^{A P}(t)$ and of A-BFT slots $M_{n}(t)$, respectively.

\section{B. Proposed ABT Scheme for WiGig Users in A-BFT}

Owing to the random time-varying environments and user movements, the user should dynamically adjust the beam transmission policies based on previous training results. Furthermore, it is desirable to utilize the training opportunities efficiently, because the training is constrained by the number of SSW frames provided from the WiGig APs, i.e., $\left|\phi_{k}^{U E}(t)\right| \leq$ $F_{n}(t)$. Therefore, we proposed the ABT scheme for WiGig users in the A-BFT interval, and the detailed procedure is illustrated in Algorithm 3. Again, the user association is uncorrelated with the beam policy. Therefore, the proposed 


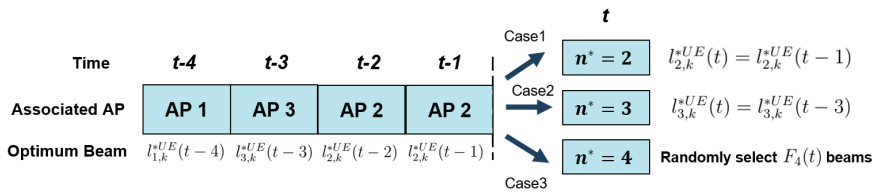

Fig. 4. Example of proposed VLF scheme. Consider user $k$ stores dataset with historical observation window size $W=4$. The previous optimum beams are given by the associated WiGig APs $\{1,3,2,2\}$, respectively.

ABT scheme operated by each WiGig user can be separately designed, including the user association and beam training policy, which are described in the following two steps.

1) User Association and Contention: Firstly, to improve the individual throughput, WiGig users will determine their own policies of association with the desired WiGig AP based on the strongest received SNR during BTI training, i.e., $n^{*}(t)=\operatorname{argmax}_{n} \gamma_{n, k, l_{n}}^{A P}(t)$. Then, the users contend for the A-BFT slot of the $n^{*}(t)$-th WiGig AP by adopting a random selection formulated as $s_{n^{*}, k}(t) \in\left[1, M_{n}(t)\right]$. Note that we adopt the random slot selection scheme in a multiple WiGig AP scenario, because the users may switch their association owing to the random environment and movements. Additionally, information gleaned from other WiGig devices in a WLAN system is mostly unknown, making it difficult for users to jointly determine the desired WiGig AP and the corresponding A-BFT slots to contend for.

2) Beam Training Policy: Without loss of generality, WiGig users may change their AP association due to the time-variant environmental conditions. Therefore, the WiGig users determine their beam training set based on the previous results of the connected AP $n^{*}(t)$. The training length of WiGig users cannot exceed the number of frames provided in each A-BFT slot, i.e., $L_{k}^{U E}(t) \leq F_{n^{*}}(t)$. To avoid wasting training resources, whilst reducing the beam alignment outage probability, the length of training set is designed to be equivalent to that of the A-BFT frame, i.e., $L_{k}^{U E}(t)=F_{n^{*}}(t)$. If the desired associated WiGig AP $n^{*}$ may be found within the previous $W$ BIs, the central beam index is determined as $l_{n^{*}, k}^{* U E}(t)=l_{n^{*}, k}^{* U E}(t-\tau)$, where $\tau \in[1, W]$, which represents that the optimal training result of the past $\tau$-th interval. Therefore, we can select the users' training index set based on the beam policy of $\phi_{k}^{U E}(t)=$ $\left\{l_{n^{*}, k}^{* U E}(t)-\left\lfloor\frac{F_{n^{*}}(t)}{2}\right\rfloor \ldots, l_{n^{*}, k}^{* U E}(t) \ldots, l_{n^{*}, k}^{* U E}(t)+\left\lfloor\frac{F_{n^{*}}(t)}{2}\right\rfloor-1\right\}$ which extends to both sides from the central beam index $l_{n^{*}, k}^{* U E}(t)$. Another WiGig AP is chosen as the candidate connection, implying that the environments randomly change, hence we randomly select $F_{n^{*}}(t)$ non-consecutive beams in the range of $\left[1, L_{k}^{U E}\right]$ for the user beam training set $\phi_{k}^{U E}(t)$. For example, as depicted in Fig. 4, let us assume that user $k$ stores historical dataset with an observation window size of $W=4$. There are three possible cases for the determination of the beam set at the $t$-th BI:

- $\quad$ Case 1: When $n^{*}=2$ is the candidate WiGig AP for user association, the user will seek the optimum beam near the current timeslot as the central beam index. Therefore, the user selects the optimum beam at the $(t-1)$-st BI, i.e., $l_{2, k}^{* U E}(t)=l_{2, k}^{* U E}(t-1)$, which is more correlated than that of time $t-2$.

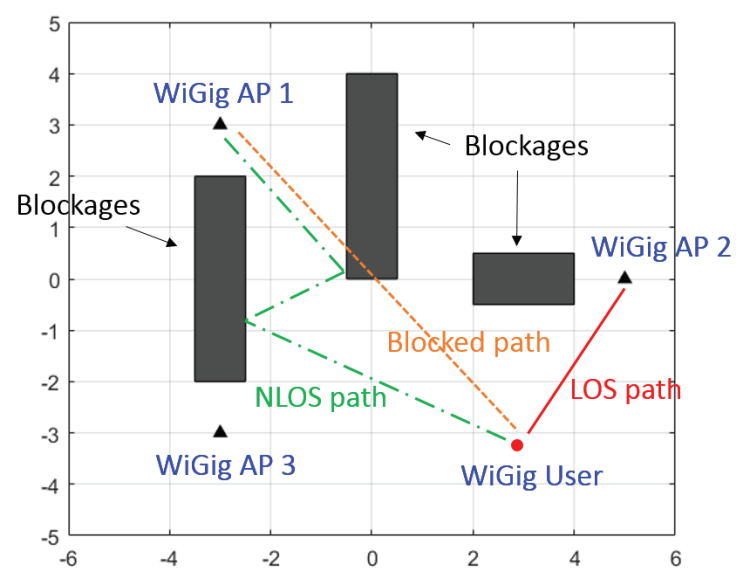

Fig. 5. Example of WiGig ray-tracing scenario considering 3 WiGig APs and 1 user along with 3 arbritrary deployed blockages. The served user potentially receives beam signals through either LOS, NLOS or blocked paths.

- $\quad$ Case 2: If the candidate AP $n^{*}=3$ is the only AP associated by user $k$ within the previous $W=4$ timeslots, the optimum beam at time $t-3$ will be chosen as the central beam, i.e., $l_{3, k}^{* U E}(t)=l_{3, k}^{* U E}(t-$ $3)$.

- $\quad$ Case 3: Consider AP $n^{*}=4$ provides the strongest signal for user association. However, user $k$ has no training results from AP 4 and will regard AP 4 as a newly associated AP. Therefore, user $k$ will randomly select $F_{4}(t)$ beams for the beam policy determination $\phi_{k}^{U E}(t)$.

We adopt circular computation of the beam indices to prevent oversized or negative values for the determination of beam training set of WiGig users, which is expressed as

$$
l= \begin{cases}\bmod \left(l, L_{k}^{U E}\right), & \text { if } l>L_{k}^{U E}, \\ \bmod \left(l+L_{k}^{U E}, L_{k}^{U E}\right), & \text { if } l<0, \\ l, & \text { otherwise, }\end{cases}
$$

where $l$ is the element of beam training index set $\phi_{k}^{U E}(t)$, and mod is the modulo operation. In our proposed ABT scheme, exhaustive search is performed when $\left|\phi_{k}^{U E}(t)\right|=F_{n^{*}}(t)$ for simultaneously preventing wastage of frames announced by the WiGig controller as well as by the APs and reduce the individual beam alignment outage probability. After the completion of beam training of WiGig APs and users, the FBs and ACKs will be exchanged. The WiGig APs report all beam outcomes, including the optimum indices and the corresponding SNRs, followed by data transmission to WiGig users.

\section{Performance Evaluation}

The system performances of our proposed CMMBT scheme and conventional methods are evaluated by simulations. We consider the general $60-\mathrm{GHz}$ WiGig based pathloss model specified in the IEEE 802.11ad/ay protocols [34] defined as

$$
P L_{\mathcal{B}}(\mathrm{dB})=A_{\mathcal{B}}+20 \log _{10} f_{c}+10 n_{\mathcal{B}} \log _{10} d_{c}+\sigma_{\mathcal{B}}
$$

where $\mathcal{B} \in\{$ LOS, NLOS $\}$ indicates the existence of either a line-of-sight (LOS) or non-line-of-sight (NLOS) path 


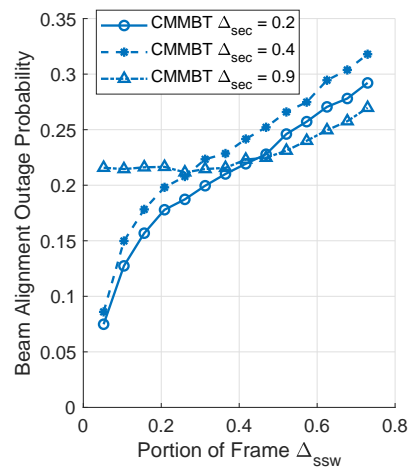

(a)

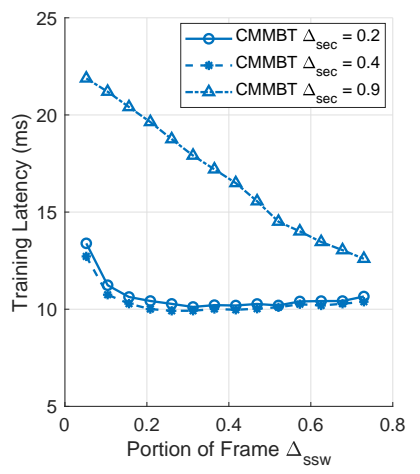

(b)

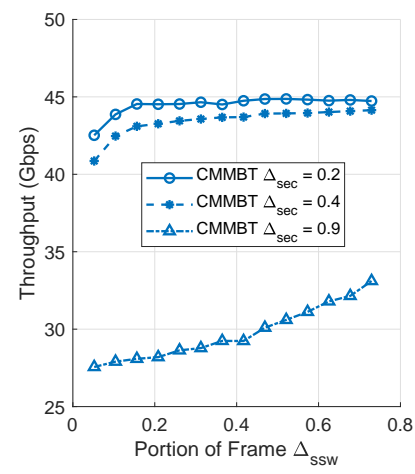

(c)

Fig. 6. Performance of varying portion differences of frames $\delta_{s s w}$ and training beams $\delta_{s e c}$ for $L_{n}^{A P}=L_{k}^{U E}=64, W=4, \delta_{b o}=0.2, \delta_{s a}=0.8$, and $M_{n}(t)=8$. (a) Beam alignment outage probability (b) training latency (c) throughput.

TABLE III

Wigig BeAm Training PARAMETERS

\begin{tabular}{ll}
\hline Parameter & Value \\
\hline \hline Channel model & Conference room [34] \\
Serving space & $12 \times 10 \times 5 \mathrm{~m}^{3}$ \\
Transmit power $P_{t}$ & $10 \mathrm{dBm}$ \\
System bandwidth $B$ & $2.16 \mathrm{GHz}$ \\
Noise power spectral density $N_{0}$ & $-174 \mathrm{dBm} / \mathrm{Hz}$ \\
Half-power beamwidth $\theta_{-3 d B}$ & $\frac{\pi}{6}$ \\
Signal decoding threshold $\gamma_{d e c}^{A P}, \gamma_{d e c}^{U E}$ & $-20 \mathrm{~dB}$ \\
Time of beacon interval $T_{B I}$ & $100 \mathrm{~ms}$ \\
Time of each sector training $T_{b m}$ & $20 \mu \mathrm{s}$ \\
Time of feedback $T_{f b}$ & $20 \mu \mathrm{s}$ \\
Time of acknowledgement $T_{a c k}$ & $20 \mu \mathrm{s}$ \\
Simulation time & $100 T_{B I}$ \\
\hline
\end{tabular}

given a specific environment. The operating frequency is $f_{c}=60 \times 10^{9} \mathrm{~Hz}$ and $d_{c}(\mathrm{~m})$ is the distance between the WiGig AP and the served user. For a conference room setting, IEEE 802.11ad/ay specifies the following parameters: pathloss constants $A_{\mathrm{LOS}}=32.5$ and $A_{\mathrm{NLOS}}=45.5$, pathloss exponents $n_{\mathrm{LOS}}=2$ and $n_{\mathrm{NLOS}}=1.4$, and shadow fading (SF) factors $\sigma_{\mathrm{LOS}}=0$ and $\sigma_{\mathrm{NLOS}}=3$ for LOS and NLOS considerations. We utilized a ray-tracing based IEEE 802.11ad/ay emulator [35] to simulate realistic wireless indoor environments and to develop our WiGig-based multi-AP multiuser beamforming training protocol. Randomly-located blockages are considered, which potentially leads to NLOS propagation conditions between the WiGig APs and users. As depicted in Fig. 5, we consider $N_{b}=3$ rectangular blocking objects with each having uniformly-distributed length and width within the range of $[2,4](\mathrm{m})$ and $[1,2](\mathrm{m})$, respectively. Furthermore, we assume the reflection factor of $N_{f}=3$ for multi-path reflections in the ray-tracing emulator. All WiGig APs are capable of providing complete coverage. For each WiGig user, we consider the random trajectory model at a velocity of $v=2(\mathrm{~m} / \mathrm{s})$. The remaining parameters are listed in Table III.

We assume that all the WiGig APs and users have the same parameters of the number of beams, i.e., we have $L_{1}^{A P}=$ $L_{2}^{A P}=\ldots=L_{N}^{A P}$ and $L_{1}^{U E}=L_{2}^{U E}=\ldots=L_{K}^{U E}$. The metrics evaluated were the successful association ratio $S A(t)$, beam alignment outage probability $B O(t)$, training latency $T_{t r}(t)$ and average throughput $R(t)$. The simulation results were divided into four parts, namely characterizing the effects of parameters, performance of global optimum, the comparison of the CMMBT scheme to the existing beam training protocols under different scenarios, and the computational complexity analysis.

\section{A. Effects of CMMBT Parameters}

In the following, we evaluate our proposed CMMBT scheme relying on different parameters, such as the portion of SSW frames and beam sectors, the historical observation window size, the threshold of beam alignment outage probability, and the number of A-BFT slots. We consider $2 \mathrm{WiGig}$ APs and 20 WiGig users for evaluating the effects of CMMBT parameters. The relationship of the CMMBT parameters with the metrics is summarized in Table IV. The notation $\lambda_{\mathcal{A}}$ indicates the portion of SSW frames $\lambda_{s s w}$ provided by the APs and beam sectors $\lambda_{\text {sec }}$ of APs, where $\mathcal{A}=\{s s w, s e c\}$.

1) Portion of SSW frames and beam sectors of APs $\lambda_{s s w}$, $\lambda_{\text {sec }}$ : : We assume that the portion has equivalent increment and decrement, i.e., $\lambda_{\mathcal{A}}^{+}=1+\Delta_{\mathcal{A}}$ and $\lambda_{\mathcal{A}}^{-}=1-\Delta_{\mathcal{A}}$, where $\mathcal{A}=\{s s w, s e c\}$. As illustrated in Fig. 6(a), we can observe that CMMBT achieves lower beam outage with a portion of $\Delta_{s s w} \leq 0.2$ because of the smoother variation of frame adjustment for WiGig users. Thus, the number of SSW frames is higher than that when $\Delta_{s s w}>0.2$, leading to a higher latency and lower throughput. Although the system satisfies the beam alignment outage constraints, CMMBT will gradually reduce the number of SSW frames provided, which results in slow convergence and degrades the system performance explicitly, where approximately $13 \mathrm{~ms}$ latency and $42 \mathrm{Gbps}$ throughput under $\Delta_{s s w} \approx 0.05$, as shown in Figs. 6(b) and 6(c), respectively. For $\Delta_{s s w}>0.2$, the alignment outage probability is increased by the fluctuating beamforming training, where WiGig users abruptly perform training relying either on a small or a large number of beam directions. However, it becomes capable of promptly reacting to environmental variations and it is more flexible in adjusting the length of the training frames, leading to a lower latency of $10 \mathrm{~ms}$ and higher throughput of $44 \mathrm{Gbps}$. We also observe that the throughput is saturated when $\Delta_{s s w} \geq 0.6$ because reduced training latency may compensate the increase in the beam 
TABLE IV

Relation Between Metrics and Parameters

\begin{tabular}{lcccc}
\hline & Portion $\lambda_{\mathcal{A}}$ & Window Size $W$ & Outage Threshold $\delta_{b o}$ & A-BFT Slots $M_{n}(t)$ \\
\hline \hline User Association & & & & $\checkmark$ \\
Alignment Outage & $\checkmark$ & $\checkmark$ & $\checkmark$ & \\
Training Latency & $\checkmark$ & $\checkmark$ & $\checkmark$ & $\checkmark$ \\
System Throughput & $\checkmark$ & $\checkmark$ & $\checkmark$ & $\checkmark$ \\
\hline
\end{tabular}

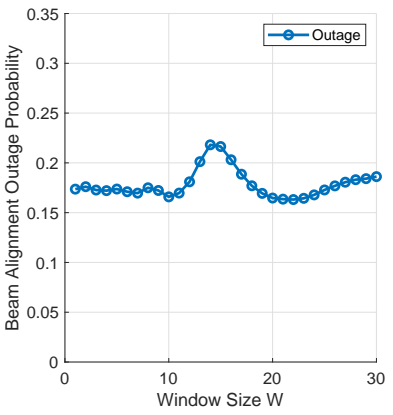

(a)

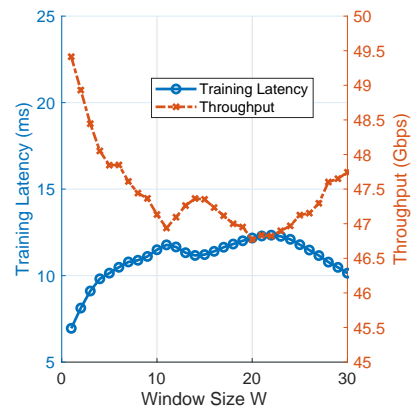

(b)

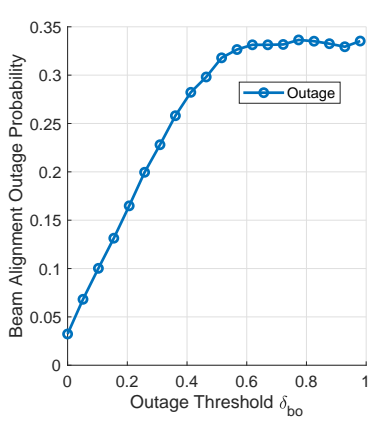

(a)

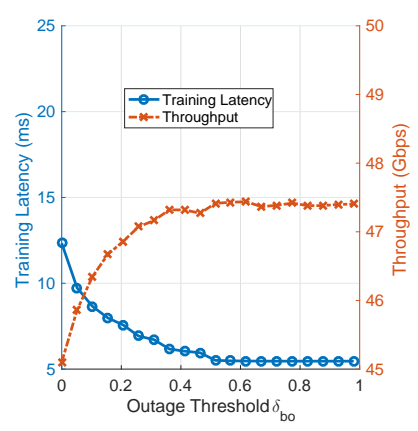

(b)

Fig. 7. Performance of different historical observation window size $W$ for $L_{n}^{A P}=L_{k}^{U E}=64, \Delta_{s e c}=\Delta_{s s w}=0.2, \delta_{b o}=0.2, \delta_{s a}=0.8$, and $M_{n}(t)=8$. (a) Beam alignment outage probability (b) training latency and throughput.

alignment outage probability. Moreover, a larger portion $\Delta_{\text {sec }}$ implies a higher fluctuation in the number of training beams of the WiGig APs. Let us now consider $\Delta_{\text {sec }}=\{0.2,0.4\}$ as shown in Fig. 6(c); we observe that CMMBT achieves slightly lower latency when $\Delta_{\text {sec }}=0.4$ compared to that for $\Delta_{\text {sec }}=0.2$. However, a higher beam alignment outage probability is achieved for $\Delta_{s e c}=0.4$, which results in a lower throughput than that for $\Delta_{s e c}=0.2$. When $\Delta_{s e c}=0.9$, the WiGig APs train their beams in sudden increases or decreases, which accordingly leads to the highest outage when $\Delta_{s s w} \leq 0.4$. Although the lowest beam outage is achieved for $\Delta_{s s w} \geq 0.4$ owing to the compensation from the frame adjustment part, its high latency results in approximately $30 \%$ throughput reduction when $\Delta_{s s w} \approx 0.75$, as shown in Fig. 6(c). In summary, $\Delta_{\text {sec }}=0.2$ and $\Delta_{s s w}=0.2$ will be adopted in Subsection IV-C for the performance comparison of our proposed CMMBT scheme.

2) Historical observation window size $W$ : We quantify the effect of using the previous $W$ historical training data for acquiring the current training policy. As depicted in Fig. 7 (a), the beam alignment outage probability slightly decreases for $W \leq 10$ observations, because the previous datasets that are not located far away from the current timeslot are highly correlated. Therefore, CMMBT adopts correlated information to obtain a better control policy for reducing the latency to approximately $7 \mathrm{~ms}$ and for attaining a high throughput of approximately $49.5 \mathrm{Gbps}$, as shown in Fig. 7(b). Under $10<W \leq 15$, insignificant historical results are utilized by CMMBT. A few beams and SSW frames are offered to compensate for the high alignment outage probability, which decreases the latency and increases the throughput. However, for $15<W \leq 22$, we can infer that the lower correlation historical dataset results in the phenomenon that more frames

Fig. 8. Performance of different beam alignment outage threshold values $\delta_{b o}$ with setting as $L_{n}^{A P}=L_{k}^{U E}=64, W=4, \Delta_{s e c}=\Delta_{s s w}=0.2$, $\delta_{s a}=0.8$, and $M_{n}(t)=8$. (a) Beam alignment outage probability (b) training latency and throughput.

are offered to the users and more beams are trained, which results in increased latency and reduced throughput. Moreover, consider a larger window size, i.e., $W>22$, most of the previous results show no correlation with the current decision. Therefore, CMMBT will only adjust a few number of training beams and frames to achieve better performances. Based on maximizing the throughput, it is appropriate to select a smaller observation window size for the proposed CMMBT scheme.

3) Threshold of beam alignment outage probability $\delta_{b o}$ :

We will evaluate the system performance by considering the influence of threshold values of beam alignment outage probability. As demonstrated in Fig. 8(a), we can observe that we have a monotonically increasing outage when $0<\delta_{b o} \leq 0.6$, which satisfies the outage threshold. Please note that CMMBT is inapplicable for $\delta_{b o}=0$ as 0 outage probability occurs when using trivial SNR threshold values, which means that all beams can be utilized for data transmission. Since the WiGig system satisfies the constraint of beam alignment outage, the WiGig APs/users intend to reduce the lengths of SSW frames and training beams, respectively, resulting in the reduction of system latency. The alignment outage potentially degrades the average received signal strength; however, the misalignment incurred allows us to have a reduced training-induced latency, which in turn results in improving the throughput. For $0.6<\delta_{b o} \leq 1$, the beam alignment outage probability has become saturated around 0.34 , the latency falls to about 5.5 ms and throughput satisfies around 47.5 Gbps. Therefore, we will select $\delta_{b o}=0.6$ as the beam alignment outage constraint to compare the performance of proposed CMMBT scheme and other benchmarks.

4) Number of A-BFT slot $M_{n}(t)$ : Because varying the threshold values of $\delta_{s a}$ can have similar effects for different numbers of A-BFT slots, we then evaluate different values 


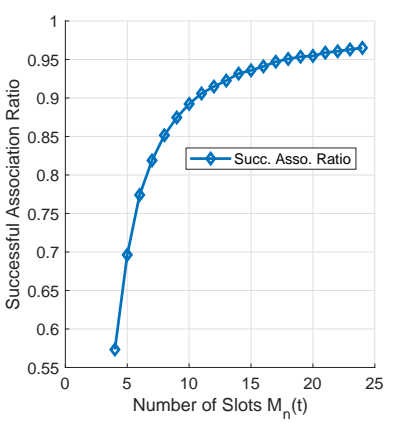

(a)

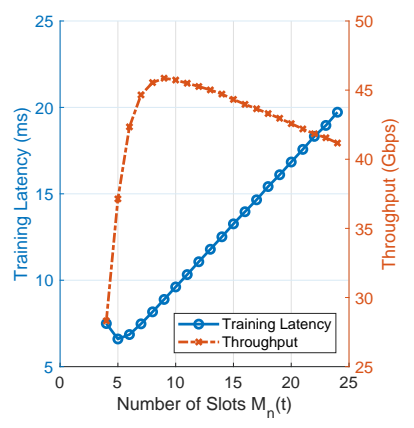

(b)
Fig. 9. Performance for different numbers of A-BFT slots $M_{n}(t)$ provided for WiGig users with setting $L_{n}^{A P}=L_{k}^{U E}=64, W=4, \Delta_{s e c}=\Delta_{s s w}=$ 0.2 , and $\delta_{b o}=0.2$. (a) Successful association ratio (b) Training latency and throughput.

of $M_{n}(t)$ instead of $\delta_{s a}$. It is intuitive from the system model (8) that the number of A-BFT slots only affects the user association but not the beam alignment. Therefore, in addition to its latency and throughput, we also characterize CMMBT in terms of its successful association ratio under different $M_{n}(t)$. We can observe from Fig. 9(a) that upon monotonically increasing the number of slots the system can support more WiGig users. Furthermore, the throughput curve of Fig. 9(a) exhibits a concave shape and it achieves the optimum of $M_{n}(t)=8$. However, an excessive number of A-BFT slots will lead to higher training latency, as shown in Fig. 9(b). With more contending slots of $M_{n}(t)>8$, the throughput is monotonically decreasing because the successful association ratio becomes saturated around 0.95 , which can not compensate high training latency of the WiGig system.

\section{B. Globally Optimal Performance}

To characterize the optimum performance, we relied on the brute-force full-search method for finding the global optimum of a computationally manageable problem by maximizing the throughput metric. However, we note that because of the exponentially escalating computational complexity of any bruteforce full-search, it cannot be implemented in practical systems. Therefore, we provide a demonstration by considering a small problem in Fig. 10 associated with $L_{n}^{A P}=L_{k}^{U E}=2$ beams, $N=2$ WiGig APs. The number of users spans from $K=2$ to $K=10$. Observe from Fig. 10 that the training latency of CMMBT at $K=10$ is about $0.4 \mathrm{~ms}$, whilst it is about $2 \mathrm{~ms}$ for the high-complexity full-search, which hence attains a higher throughout of about $18 \mathrm{Gbps}$ for $K=10$ than that of the CMMBT saturating around $15 \mathrm{Gbps}$. The throughput of CMMBT starts to saturate around $K=5$, while that of the full search around $K=8$, which is a benefit of the higher SNR attained by full search. By contrast, a smaller fraction of beams is tested by the more practical CMMBT scheme, which strikes a compelling throughput, latency and complexity trade-off in practical implementations.

\section{Performance Comparison for Proposed CMMBT Scheme}

We compare the proposed CMMBT scheme to two benchmarks (BMs), which are referred to as the time aligned beam-

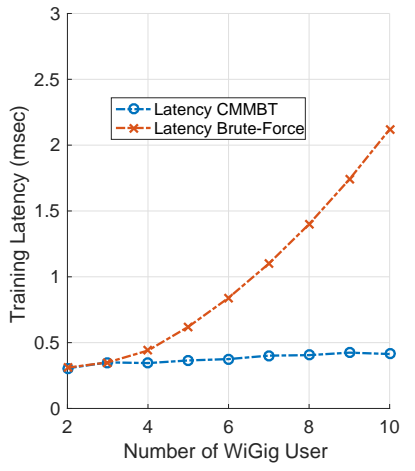

(a)

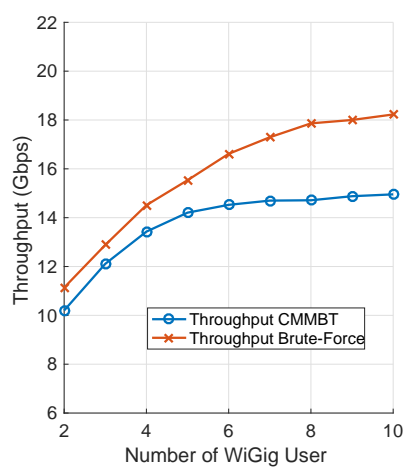

(b)
Fig. 10. Performance for global optimum of (a) training latency and (b) throughput considering the small size problem with CMMBT setting as $N=$ $2, L_{n}^{A P}=L_{k}^{U E}=2, \Delta_{s e c}=\Delta_{s s w}=0.2, M_{n}(t)=8, W=4$, $\delta_{b o}=0.6, \delta_{s a}=0.8$. Note that latency is calculated based on the number of beams multiplied by duration of frame per beam.

forming training (TABT) protocol of [31] and the fixed framing mechanism with exhaustive beam training (FixExh) [4]. The benchmark TABT adopts time-division multiplexing (TDM) based framing along with scheduled and contentionfree beam training for WiGig APs and users. Moreover, complete alignment is performed in TABT by utilizing the exhaustive beam search method. For FixExh, the contentionbased training protocol is executed, as illustrated in Fig. 1 and 2. However, the number of SSW frames and of A-BFT slots are fixed in FixExh as the maximum available support. Exhaustive search is also adopted as its beamforming training, which can be considered as the worst case of CMMBT. Based on the proposed system model, the latency of TABT can be expressed as

$$
T^{T A B T}=T_{b m} \sum_{n=1}^{N}\left[L_{n}^{A P}+L_{k}^{U E}\right]+N K,
$$

and the latency of FixExh is given by

$$
T^{\mathrm{FixExh}}=T_{b m} \sum_{n=1}^{N} L_{n}^{A P}+\sum_{n=1}^{N}\left[T_{b m} M_{n} F_{n}+\left(T_{f b}+T_{a c k}\right) M_{n}\right] .
$$

Based on our parametric study in the previous subsection, we select $\Delta_{s e c}=\Delta_{s s w}=0.2, \delta_{b o}=0.6, \delta_{s a}=0.8, M_{n}=8$ and $W=4$ as the parameter settings for our performance comparisons. In the following section, we compare successful association ratio, beam alignment outage probability, training latency, and throughput for different numbers of beams and WiGig users.

1) Effects of the Number of Beams: As shown in Fig. 11(a), we can observe that TABT achieves full association for every WiGig user due to its scheduled beamforming training protocol with an arbitrary number of WiGig APs. However, for the contention-based scheme in CMMBT and FixExh, the successful association ratio is affected by the number of serving WiGig APs. It is observed that it can support more connections of WiGig users upon increasing the number of WiGig APs quantitatively, 93\% of WiGig users become associated with one of the $N=4 \mathrm{WiGig}$ APs. Moreover, as 


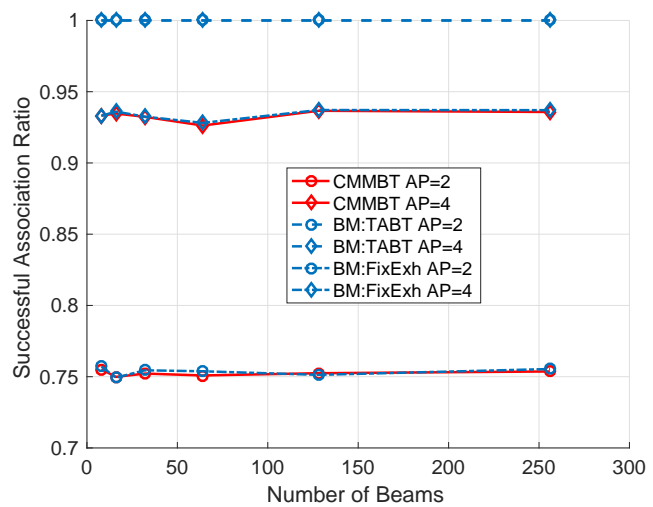

(a)

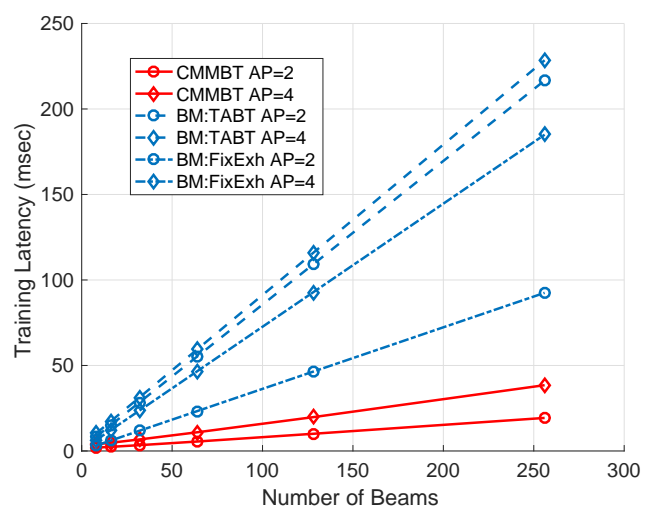

(c)

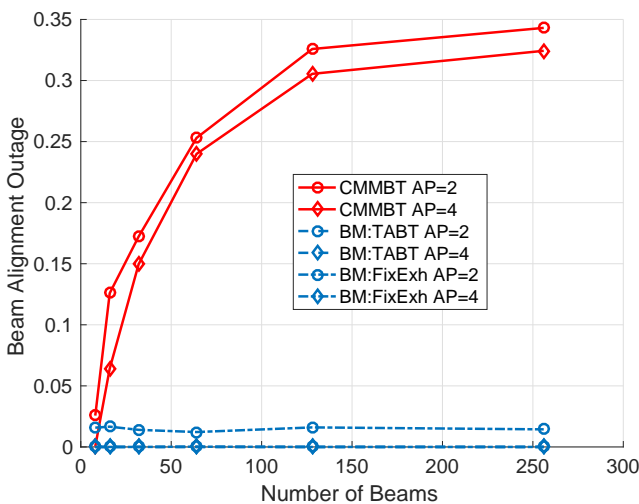

(b)

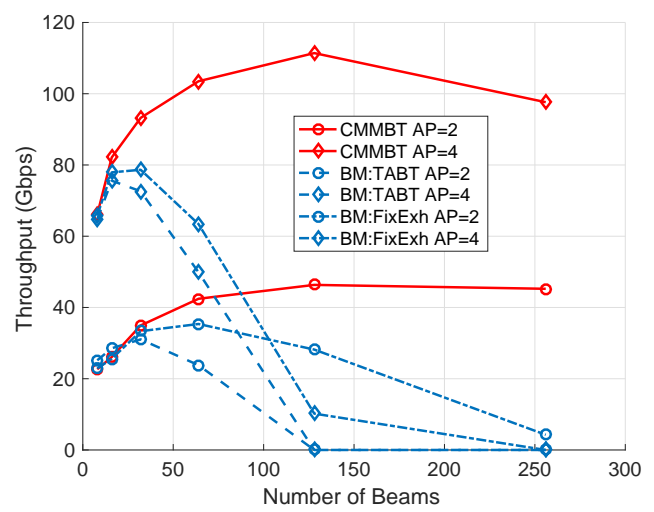

(d)

Fig. 11. Simulation results of CMMBT scheme compared with TABT and FixExh methods with different numbers of beams $L_{n}^{A P}=L_{k}^{U E}=$ $\{8,16,32,64,128,256\}$ for $K=20$ WiGig users. (a) Successful association ratio, (b) beam alignment outage, (c) training latency, (d) throughput.

shown in Fig. 11(b), the number of beams has little influence on user association, which can also be deduced from the formulation of (8). Since exhaustive beamforming training is adopted in TABT and FixExh, the number of beams has no impacts on the beam alignment outage probability. However, our proposed CMMBT achieves an outage probability as around 0.35 for 256 beams, which satisfies the beam outage constraint, i.e., $\delta_{b o}=0.6$. Since we can tolerate a certain maximum beam alignment outage probability, CMMBT has the option of reducing the length of beam training for the sake of reducing the latency. As seen in Fig. 11(c), the proposed CMMBT has the lowest latency owing to its flexible adjustment of the number of frames, slots, and beams. Observe that since the training structure is confined to the maximum available $M_{n}$ A-BFT slots for the FixExh method, its highest latency mainly arises from the enormous number of training beams of the WiGig APs.

Furthermore, it can be deduced from Fig. 11(d) that the throughput exhibits a concave shape for all schemes due to the trade-off between latency, beam alignment outage, and beam gains, which imposes opposing trends. The trade-off comes from the competition of policies of beams and frames. Under a smaller number of training beams of $L \leq 128$, the throughput increases due to domination of ultra-low latency coming from a few number of training frames; On the other hand, $L>128$, narrower beams require more SSW training frames, which leads to a higher training latency. Additionally, even with high- gain of beams, the throughput decreases due to excessive nonaligned beams under insufficient training opportunities. The proposed CMMBT scheme achieves the highest throughput of $110 \mathrm{Gbps}$ supported by $N=4$ WiGig APs with 128 beams, and around $47 \mathrm{Gbps}$ by $N=2$ APs with 128 beams. For FixExh with less than 128 beams, we observe that the scenario of $N=4$ WiGig APs outperforms that with $N=2$ APs due to the diversity gain of more beams offered by the different APs. However, for more than 128 beams, the beam training is overwhelmed which reduces the throughput when adopting TABT and FixExh. Therefore, little time remains for downlink transmission having insufficient training SSW frames for 256 beams, regardless of the number of WiGig APs employed. In the worst case, the WiGig system has zero throughput by adopting the TABT scheme for more than 128 beams. The proposed CMMBT circumvents this problem by flexibly adjusting the length of the training frames and the corresponding beam directions at a modestly increased alignment outage probability, which still supports rates of 45 and $100 \mathrm{Gbps}$ throughput under $N=2$ and $N=4 \mathrm{WiGig}$ APs equipped with 256 beams, respectively. Our CMMBT benefits from high-gain beams and reduced training overheads, hence supports directional transmission at the highest throughput among all schemes.

2) Effect of the Number of WiGig Users: In this subsection, performance comparisons are conducted for different numbers of WiGig users and $N=2$ WiGig APs. As illustrated 


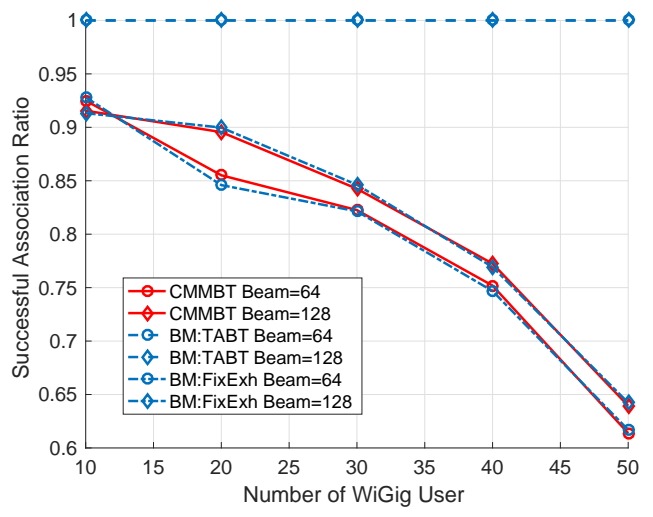

(a)

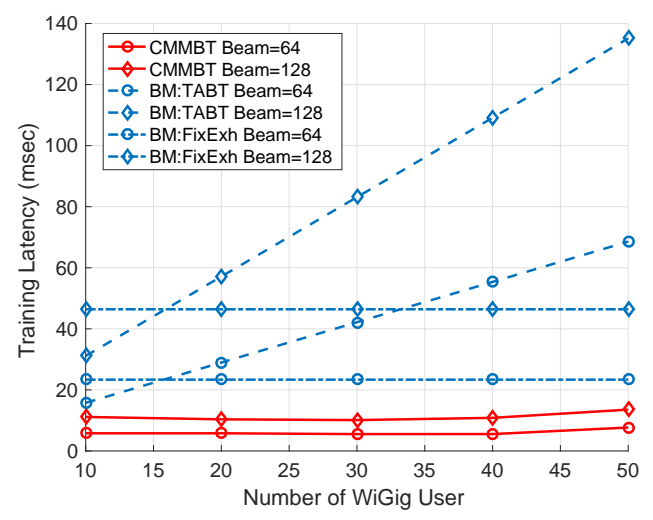

(c)

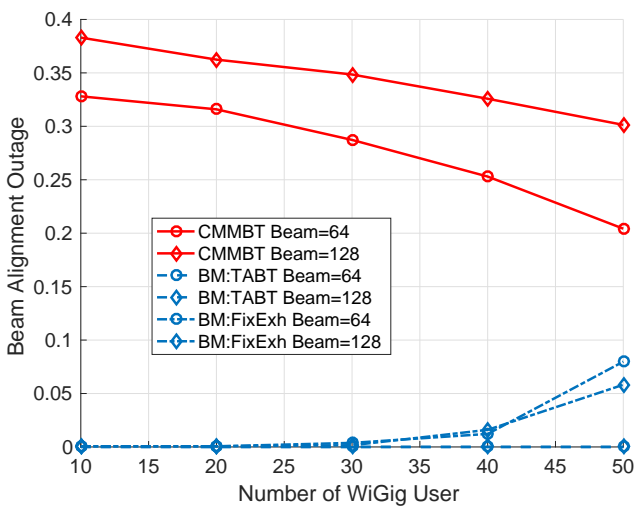

(b)

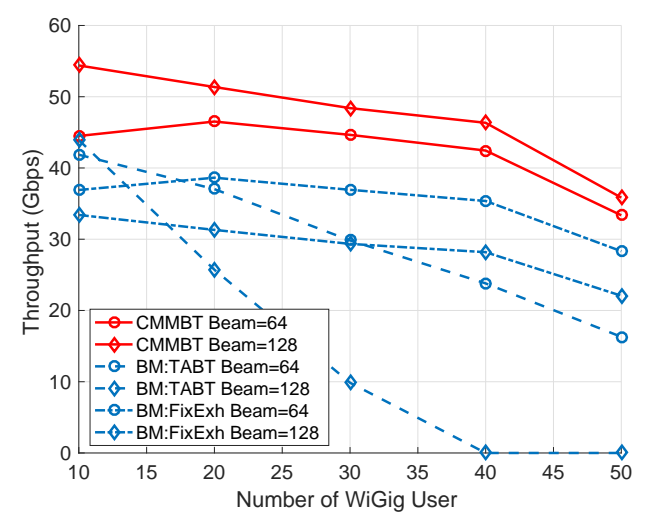

(d)

Fig. 12. Simulation results of the CMMBT scheme compared with the TABT and FixExh methods with different numbers of WiGig users $K=$ $\{10,20,30,40,50\}$ served by $N=2$ WiGig APs. (a) Successful association ratio, (b) beam alignment outage, (c) training latency, (d) throughput.

in Fig. 12(a), the benchmark TABT training mechanism is capable of achieving a full association ratio, and the number of beams will have little impact on the association, as discussed in the previous subsection. Under the association constraint of $\delta_{s a}=0.8$, the WiGig APs utilizing CMMBT and FixExh can only support at most about $30 \mathrm{WiGig}$ users. This is because for more than 30 users, the WiGig APs cannot satisfy the predefined threshold and instead they focus on the beam alignment of the associated devices. We can observe from Fig. 12(b) that CMMBT exhibits a gradually reduced beam alignment outage probability, which is reduced from 0.38 for $K=10$ users to 0.3 for $K=50$ users when 128 beams are employed. This is because the CMMBT scheme sacrifices some A-BFT slots by providing more SSW frames for the beam alignment process. Although the FixExh supports the maximum number of contention and training opportunities, its relatively rigid non-adjustable mechanism and crowded scenarios will degrade the beam alignment. Hence, the outage probability increases from 0 to 0.08 for $K=10$ and $K=20 \mathrm{WiGig}$ users, respectively. However, TABT using a training scheme identical to that of FixExh achieves zero beam alignment outage since all the beam directions will be scheduled and trained without any contention. However, the training latency of TABT linearly increases upon serving more WiGig users. As shown in Fig. 12(c), under the newly proposed transmission structure for the multiple WiGig APs and users, the proposed CMMBT adjusts the number of A-BFT slots, SSW frames, or the length of the training beams to maintain near-constant overhead for different number of WiGig users. It is worth mentioning that we can regard the performance of the FixExh scheme as our bounds due to the maximum exploitation of all the frames and beam resources under an identically designed frame structure.

Observe from Fig. 12(d) that the throughput is reduced upon serving more WiGig users among for the schemes, but for different reasons. Although full association and beam training is achieved by TABT, its high training latency leads to the lowest throughput in most cases. However, both FixExh and CMMBT are affected by a low successful association ratio. The unassociated WiGig users may have optimal beam conditions, which provides selection diversity gain. Accordingly, having few selections of beams degrades the throughput owing to having fewer connected WiGig users. Additionally, the beam alignment outage of FixExh further degrades the downlink transmissions. Note that when serving $K=10$ WiGig users, TABT achieves a higher throughput than FixExh as a benefit of its lower overhead. In other words, under a small number of users, fewer frames and slots are utilized for scheduled training in TABT compared to a fully loaded FixExh scheme. It is also worth mentioning that CMMBT exhibits the opposite trend, when considering different number of beams, explicitly the throughput of 128 beams is higher than that of 64 beams. In summary, our proposed CMMBT imposes the lowest training latency and yet achieves the highest throughput at an acceptable degradation arising from beam alignment outage 
TABLE V

SCHEME COMPLEXITY AND COMPUTATION TIME

\begin{tabular}{|l|r|}
\hline Beam Training Schemes & Computational Complexity \\
\hline \hline Proposed CMMBT & $\mathcal{O}(W)$ \\
\hline BM 1: TABT & $\mathcal{O}(N K)$ \\
\hline BM 2: FixExh & $\mathcal{O}(1)$ \\
\hline Brute-Force & $\mathcal{O}\left(N^{K} F M 2^{L^{A P}} 2^{L^{U E}}\right)$ \\
\hline
\end{tabular}

compared to the TABT and FixExh benchmarks.

\section{Complexity Analysis}

The complexity of the proposed CMMBT as well as of the benchmarks and of the brute-force method is summarized in Table V. The global optimum of problem (10) can be obtained by employing the brute-force method, which is impractical due to its ultra-high complexity order of $\mathcal{O}\left(N^{K} F M 2^{L^{A P}} 2^{L^{U E}}\right)$. By contrast, for our proposed CMMBT, no iterations are required in VLF because the beam training policies of the WiGig controller and of the APs are only decided by the thresholds and the historical beam results, leading to "single-shot" decision making. Furthermore, the proposed ABT scheme is individually operated in all WiGig user devices. As stated in ABT, it only requires $W$ iterations to obtain its optimum training beam set. The complexity of our proposed CMMBT scheme is on the order of $\mathcal{O}(W)$, which exhibits polynomial complexity increase with $W$, hence it is readily implementable on WiGig-based platforms. However, the complexity of TABT is $\mathcal{O}(N K)$, which is much higher for its time-division-based scheduled training in the fact of an enormous number of WiGig connections. When considering ultra-dense deployment, TABT is not suitable due to its high complexity. Although FixExh having a fixed structure and exhaustive beam training has a complexity order of $\mathcal{O}(1)$, it limits the system performance due to its inflexibility. Considering ultra-fast transmissions relying on pencil-beams such as $1^{\circ}$ beamwidth, the training latency becomes excessive, hence there will be insufficient time for supporting downlink data transfer. To sum up, our CMMBT scheme relying on our novel WiGig-based beam training frame structure outperforms the existing schemes and protocols at an acceptable computation complexity.

\section{CONCLUSiOnS}

We have designed a new transmission frame structure for multiple WiGig APs and multiple users, which is compatible with the current IEEE 802.11ad/ay specifications. We have maximized the system throughput by jointly considering WiGig user association, beam alignment outage probability and beam training latency. Based on the problem formulated, we proposed the CMMBT scheme for flexibly adjusting the length of training frames and slots as well as the beam policies of individual WiGig APs and users. The impact of parameters was evaluated through ray-tracing based simulations. The performance results demonstrated that at a modest successful association ratio and beam alignment outage probability, our proposed CMMBT scheme outperforms the existing TABT training protocols and FixExh schemes both in terms of its training latency and throughput. Finally, our proposed CMMBT scheme has a modest complexity.

\section{REFERENCES}

[1] A. Osseiran, F. Boccardi, V. Braun et al., "Scenarios for 5G Mobile and Wireless Communications: The Vision of the METIS Project," IEEE Communications Magazine, vol. 52, no. 5, pp. 26-35, May 2014.

[2] L. Wei, R. Q. Hu, Y. Qian et al., "Key Elements to Enable Millimeter Wave Communications for 5G Wireless Systems," IEEE Wireless Communications, vol. 21, no. 6, pp. 136-143, Dec. 2014.

[3] M. Giordani, M. Polese, A. Roy et al., "A Tutorial on Beam Management for 3GPP NR at mmWave Frequencies," IEEE Communications Surveys Tutorials, vol. 21, no. 1, pp. 173-196, 1st Quarter 2019.

[4] T. Nitsche, C. Cordeiro, A. B. Flores et al., "IEEE 802.11ad: Directional $60 \mathrm{GHz}$ Communication for Multi-Gigabit-per-Second Wi-Fi," IEEE Communications Magazine, vol. 52, no. 12, pp. 132-141, Dec. 2014.

[5] P. Zhou, K. Cheng, X. Han et al., "IEEE 802.11ay-Based mmWave WLANs: Design Challenges and Solutions," IEEE Communications Surveys Tutorials, vol. 20, no. 3, pp. 1654-1681, 3rd Quarter 2018.

[6] C. J. Hansen, "WiGig: Multi-Gigabit Wireless Communications in the $60 \mathrm{GHz}$ Band," IEEE Wireless Communications, vol. 18, no. 6, pp. 6-7, Dec. 2011.

[7] L. H. Shen, Y. C. Chen, and K. T. Feng, "Design and Analysis of MultiUser Association and Beam Training Schemes for Millimeter Wave based WLANs," IEEE Transactions on Vehicular Technology, pp. 1-1, 2020.

[8] I. A. Hemadeh, K. Satyanarayana, M. El-Hajjar et al., "Millimeter-Wave Communications: Physical Channel Models, Design Considerations, Antenna Constructions, and Link-Budget," IEEE Communications Surveys Tutorials, vol. 20, no. 2, pp. 870-913, 2nd Quarter 2018.

[9] H. Shokri-Ghadikolaei, C. Fischione, G. Fodor et al., "Millimeter Wave Cellular Networks: A MAC Layer Perspective," IEEE Transactions on Communications, vol. 63, no. 10, pp. 3437-3458, Oct. 2015.

[10] L. H. Shen and K. T. Feng, "Mobility-Aware Subband and Beam Resource Allocation Schemes for Millimeter Wave Wireless Networks," IEEE Transactions on Vehicular Technology, pp. 1-1, 2020.

[11] S. Han, C. I, Z. Xu et al., "Large-Scale Antenna Systems with Hybrid Analog and Digital Beamforming for Millimeter Wave 5G," IEEE Communications Magazine, vol. 53, no. 1, pp. 186-194, Jan. 2015.

[12] A. Li and C. Masouros, "Energy-Efficient SWIPT: From Fully Digital to Hybrid AnalogDigital Beamforming," IEEE Transactions on Vehicular Technology, vol. 67, no. 4, pp. 3390-3405, Apr. 2018.

[13] A. Abrardo, G. Fodor, and M. Moretti, "Distributed Digital and Hybrid Beamforming Schemes With MMSE-SIC Receivers for the MIMO Interference Channel," IEEE Transactions on Vehicular Technology, vol. 68, no. 7, pp. 6790-6804, Jul. 2019.

[14] A. F. Molisch, V. V. Ratnam, S. Han et al., "Hybrid Beamforming for Massive MIMO: A Survey," IEEE Communications Magazine, vol. 55, no. 9, pp. 134-141, Sep. 2017.

[15] K. T. Feng, L. H. Shen, C. Y. Li, P. T. Huang, S. H. Wu, L. C. Wang, Y. B. Lin, and M. C. F. Chang, "3D On-Demand Flying Mobile Communication for Millimeter Wave Heterogeneous Networks," IEEE Network, pp. 1-7, 2020.

[16] T. Nadeem, "Analysis and Enhancements for IEEE 802.11 Networks Using Directional Antenna With Opportunistic Mechanisms," IEEE Transactions on Vehicular Technology, vol. 59, no. 6, pp. 3012-3024, Jul. 2010.

[17] H. Shokri-Ghadikolaei, L. Gkatzikis, and C. Fischione, "BeamSearching and Transmission Scheduling in Millimeter Wave Communications," in Proc. of IEEE International Conference on Communications (ICC), Jun. 2015, pp. 1292-1297.

[18] J. Fan, L. Han, X. Luo et al., "Beamwidth Design for Beam Scanning in Millimeter-Wave Cellular Networks," IEEE Transactions on Vehicular Technology, Nov. 2019.

[19] D. De Donno, J. Palacios, and J. Widmer, "Millimeter-Wave Beam Training Acceleration Through Low-Complexity Hybrid Transceivers," IEEE Transactions on Wireless Communications, vol. 16, no. 6, pp. 3646-3660, Jun. 2017.

[20] B. Li, Z. Zhou, H. Zhang et al., "Efficient Beamforming Training for $60-\mathrm{GHz}$ Millimeter-Wave Communications: A Novel Numerical Optimization Framework," IEEE Transactions on Vehicular Technology, vol. 63, no. 2, pp. 703-717, Feb. 2014.

[21] J. Kim and A. F. Molisch, "Fast Millimeter-Wave Beam Training with Receive Beamforming," Journal of Communications and Networks, vol. 16, no. 5, pp. 512-522, Oct. 2014.

[22] L. H. Shen, Y. C. Chen, and K. T. Feng, "Mobility-Aware Fast Beam Training Scheme for IEEE 802.11ad/ay Wireless Systems," in Proc. of IEEE Wireless Communications and Networking Conference (WCNC), Apr. 2018, pp. 1-6. 
[23] Q. Xue, X. Fang, M. Xiao et al., "Multiuser Millimeter Wave Communications With Nonorthogonal Beams," IEEE Transactions on Vehicular Technology, vol. 66, no. 7, pp. 5675-5688, Jul. 2017.

[24] A. Biason and M. Zorzi, "Multicast via Point to Multipoint Transmissions in Directional 5G mmWave Communications," IEEE Communications Magazine, vol. 57, no. 2, pp. 88-94, Feb. 2019.

[25] A. Akhtar and S. C. Ergen, "Efficient Network Level Beamforming Training for IEEE 802.11ad WLANs," in Proc. of Symposium on Performance Evaluation of Computer and Telecommunication Systems (SPECTS), Jul. 2015, pp. 1-6.

[26] P. Zhou, X. Fang, Y. Fang et al., "Enhanced Random Access and Beam Training for Millimeter Wave Wireless Local Networks With High User Density," IEEE Transactions on Wireless Communications, vol. 16 , no. 12, pp. 7760-7773, Dec. 2017.

[27] Y. C. Chen, L. H. Shen, and K. T. Feng, "Enhanced Multi-User Beamforming Protocol for Millimeter Wave Wireless Local Area Networks," in Proc. of IEEE Wireless Communications and Networking Conference (WCNC), Apr. 2018, pp. 1-6.

[28] M. Kim, T. Ropitault, S. Lee et al., "Efficient MU-MIMO Beamforming Protocol for IEEE 802.11ay WLANs," IEEE Communications Letters, vol. 23, no. 1, pp. 144-147, Jan. 2019.

[29] E. M. Mohamed, H. Kusano, K. Sakaguchi et al., "WiFi Assisted MultiWiGig AP Coordination for Future Multi-Gbps WLANs," in Proc. of IEEE Personal, Indoor, and Mobile Radio Communications (PIMRC), Aug. 2015, pp. 1980-1984.

[30] E. M. Mohamed, K. Sakaguchi, and S. Sampei, "Wi-Fi Coordinated WiGig Concurrent Transmissions in Random Access Scenarios," IEEE Transactions on Vehicular Technology, vol. 66, no. 11, pp. 1035710371, Nov. 2017.

[31] S. Kei, M. E. Mahmoud, K. Hideyuki et al., "Millimeter-Wave Wireless LAN and Its Extension Toward 5G Heterogeneous Networks," IEICE Transactions on Communications, vol. 98, no. 10, pp. 1932-1948, 2015.

[32] P. Zhou, X. Fang, X. Wang et al., "Deep Learning-Based Beam Management and Interference Coordination in Dense mmWave Networks,' IEEE Transactions on Vehicular Technology, vol. 68, no. 1, pp. 592-603, Jan. 2019.

[33] A.-S. Shadi, B. Koichiro, B. Raja et al., "PHY/MAC Complete Proposal Specification," IEEE Document 802.11-10/0433r2, May 2010.

[34] A. Maltsev, V. Erceg, E. Perahia et al., "Channel Models for $60 \mathrm{GHz}$ WLAN Systems," IEEE Document 802.11-09/0334r8, May 2010.

[35] D. Steinmetzer, J. Classen, and M. Hollick, "mmTrace: Modeling Millimeter-Wave Indoor Propagation with Image-based Ray-Tracing," in Proc. of IEEE Conference on Computer Communications Workshops (INFOCOM WKSHPS), Apr. 2016, pp. 429-434.

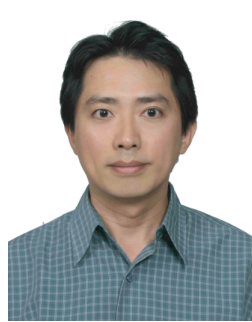

Kai-Ten Feng received the B.S. degree from the National Taiwan University, Taipei, Taiwan, in 1992, the M.S. degree from the University of Michigan, Ann Arbor, in 1996, and the Ph.D. degree from the University of California, Berkeley, in 2000. Since August 2011, he has been a full Professor with the Department of Electrical and Computer Engineering, National Chiao Tung University (NCTU), Hsinchu, Taiwan, where he was an Associate Professor and Assistant Professor from August 2007 to July 2011 and from February 2003 to July 2007, respectively. He served as the Associated Dean of Electrical and Computer Engineering College at NCTU starting from February 2017. From July 2009 to March 2010, he was a Visiting Research Fellow with the Department of Electrical and Computer Engineering, University of California at Davis. Between 2000 and 2003, he was an In-Vehicle Development Manager/Senior Technologist with OnStar Corporation, a subsidiary of General Motors Corporation, where he worked on the design of future Telematics platforms and in-vehicle networks. His current research interests include broadband wireless networks, cooperative and cognitive networks, smart phone and embedded system designs, wireless location technologies, and intelligent transportation systems.

Dr. Feng received the Best Paper Award from the Spring 2006 IEEE Vehicular Technology Conference, which ranked his paper first among the 615 accepted papers. He also received the Outstanding Youth Electrical Engineer Award in 2007 from the Chinese Institute of Electrical Engineering, and the Distinguished Researcher Award from NCTU in 2008, 2010, and 2011. He has been serving as the technical advisor for IEEE-HKN honor society and National Academy of Engineering (NAE) grand challenges scholars program (GCSP) at NCTU since 2018. He has also served on the technical program committees in various international conferences.

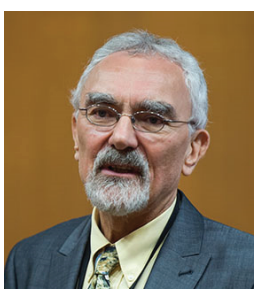

Lajos Hanzo (http://www-mobile.ecs.soton.ac.uk, https://en.wikipedia.org/wiki/Lajos_Hanzo) (FIEEE'04, Fellow of the Royal Academy of Engineering F(REng), of the IET and of EURASIP), received his Master degree and Doctorate in 1976 and 1983, respectively from the Technical University (TU) of Budapest. He was also awarded the Doctor of Sciences (DSc) degree by the University of Southampton (2004) and Honorary Doctorates by the TU of Budapest (2009) and by the University of Edinburgh (2015). He is a Foreign Member of the Hungarian Academy of Sciences and a former Editor-in-Chief of the IEEE Press. He has served several terms as Governor of both IEEE ComSoc and of VTS. He has published 1900+ contributions at IEEE Xplore, 19 Wiley-IEEE Press books and has helped the fast-track career of $123 \mathrm{PhD}$ students. Over 40 of them are Professors at various stages of their careers in academia and many of them are leading scientists in the wireless industry.

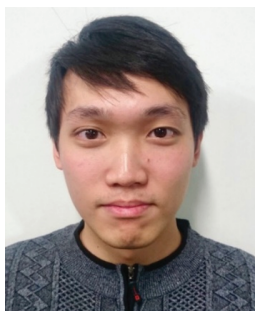

Li-Hsiang Shen received the B.S. degree in 2015 from the department of Electrical and Computer Engineering, National Chiao Tung University (NCTU), Hsinchu, Taiwan. Since 2015, he has been pursuing his Ph.D. degree in the Institute of Communication Engineering, NCTU, Hsinchu, Taiwan. In 2018 and 2019 , he was a visiting scholar of Next Generation Wireless (NGW) research group of electrical and computer engineering at the University of Southampton at Southampton (Soton), UK. His research interests include millimeter wave interference management and resource allocation design, mobility-aware beam control and training protocol design for wireless local area networks (WLANs), and 5G new radio (5G-NR) wireless communication networks. 